\title{
Almost-synchronous solutions for mutually coupled excitatory neurons
}

\author{
Amitabha Bose ${ }^{*, a, 1}$, Nancy Kopell ${ }^{\mathrm{b}, 2}$, David Terman ${ }^{\mathrm{c}, 3}$ \\ a Department of Mathematical Sciences, Center for Applied Mathematics and Statistics, New Jersey Institute of Technology, Newark, NJ \\ 07102, USA \\ b Department of Mathematics, Center for BioDynamics, Boston University, Boston, MA 02215, USA \\ c Department of Mathematics, Ohio State University, Columbus, OH 43210, USA
}

Received 20 May 1999; received in revised form 15 October 1999; accepted 15 November 1999

Communicated by J.P. Keener

\begin{abstract}
We consider synchronization in a pair of neurons described by voltage-gated conductance equations and coupled by mutual excitation. Our model neurons have three time scales: the very fast transition between active and inactive states; an intermediate scale during the active portion of a cell's trajectory; and the slowest during the inter-burst interval. We show that the interplay of time scales can lead to stable "almost-synchronous" solutions in which the jumps between active and inactive states of the two cells happen with a time difference that is a small fraction of the total period of the coupled system. Furthermore, modulation of parameters not affecting time scales can change the stable solution from almost-synchronous to synchronous. We use a geometric analysis that enables us to identify the parts of the trajectories over which the interactions move the coupled trajectory away from synchrony, the parameters responsible for this phenomenon and how the distance from synchrony depends on the time scales and can be modulated. (c) 2000 Elsevier Science B.V. All rights reserved.
\end{abstract}

Keywords: Oscillations; Almost-synchrony; Excitation

\section{Introduction}

Networks of neurons have many different time scales, both intrinsic and synaptic. Though much research has been done on how the interaction of these time scales affects the ability of a network to synchronize $[1-5,7-9,12,13,15,16$, 18,21-23] surprising effects continue to emerge. The work of the last few years had shown that a previous and widely held view that excitation is synchronizing and inhibition is desynchronizing is not generally true. Indeed, in some instances $[1-3,7-9,15,18,22,23]$ the opposite is true. However, the newer view that fast excitation is

\footnotetext{
* Corresponding author.

E-mail addresses: bose@m.njit.edu (A. Bose),nk@math.bu.edu (N. Kopell), terman@math.ohio-state.edu (D. Terman)

${ }^{1}$ Supported in part by NSF grant DMS-9973230 and NJIT grant 421540.

${ }^{2}$ Supported in part by NSF grant DMS-9706694 and NIH grant MH47150.

${ }^{3}$ Supported in part by NSF grant DMS-9802339.
} 
generally desynchronizing is also an oversimplification. For example, even if cells do not wholly synchronize under excitatory coupling, the robust outcome of the network dynamics may be "almost-synchronous" solutions $[12,13]$.

Using a reduced version of a model of Traub and Miles [20] with excitatory synapses, Pinsky and Rinzel [13] showed in simulations that there could be a stable state close to synchrony when the fully synchronized state was unstable. Pinsky [12] analyzed a non-biophysical caricature of such a system and produced an "almostsynchronous" solution. In related work van Vreeswijk et al. [22] analyzed a network of integrate and fire neurons, using excitatory synapses with a prescribed time course for the Excitatory Post-Synaptic Potential of the form $g \alpha^{2} t \mathrm{e}^{-\alpha t}$; they found that for large $\alpha$, there are stable non-synchronous solutions that approach synchrony as $1 / \alpha \rightarrow 0$.

This paper revisits the existence and stability of almost-synchronous solutions, this time in the context of conductance-based neurons, in the spirit of, but simpler than, Pinsky and Rinzel [13]. The goal is to understand from the biophysical time scales and parameters what gives rise to the destabilization of the synchronous solution, and what allows a nearby solution to be stable. In the process, we can see how far from synchrony this new solution is, and how this can be modulated.

In conductance-based models, there can be many time scales associated with the kinetics of different currents [14]. By changing which currents are expressed in the system, the rise time of the action potential, the time over which the system has high voltage, and the time between action potentials can all be modulated independently. In this paper, we are considering the case in which the length of the activated state is intermediate between the (short) rise time and the (longer) time between action potentials. Such a situation corresponds to a spike produced by calcium currents (which are slower to decay than spikes caused by standard sodium currents) or as a caricature of the envelope of a short burst of sodium spikes.

In this parameter regime, there are three natural time scales in the problem. The rate constants for these are denoted $\mathrm{O}(1), \mathrm{O}(\gamma), \mathrm{O}(\epsilon)$ with $\epsilon \ll \gamma \ll 1$. The synaptic currents are modeled as functions of the presynaptic voltage, with no further time scales, i.e. the synapses activate and inactivate fast as the presynaptic voltage changes.

We show that the intermediate time scale leads to almost-synchronous solutions, i.e. trajectories for the coupled pair in which the times of arrival at the threshold for firing differ by an $O(\epsilon)$ fraction of the period. The analysis involves an interplay of space and time scales, with analysis of spatial separation at different parts of the trajectory used to infer temporal differences and vice versa.

Our methods are those of geometric singular perturbation theory, extended to deal with more than two time scales. In standard two-time scale analysis, the jump between slow processes is instantaneous in the singular limit. In our setting, part of the jump, the flow during the time a neuron is active, takes place on the intermediate time scale. We show that in the early part of the active state, the dynamics pushes apart cells with sufficiently nearby initial conditions, but bring together cells whose initial conditions are further apart. The two sets of estimates provide bounds for the almost-synchronous solution in terms of the time scales.

In addition to analytical work aimed at dissecting out the origins of almost-synchronous solutions, we provide a numerical section. This section gives numerical evidence for the variety of phenomena uncovered by the analysis. It also uses the arguments of the previous work to discuss, and give numerical evidence for, the modulability of the system by parameters not connected to time scales.

The outline of the paper is as follows. In Section 2, we give the equations for the full problem and also derive relevant sets of reduced equations (i.e. $\epsilon=0$ ) which govern the behavior of the cells during different parts of their trajectories. In Section 3, we analyze the reduced equations to produce a singular almost-synchronous solution. The analysis in this section focuses on how the relative positions of intrinsic and synaptic thresholds, together with the intermediate time scale conspire to produce different types of almost-synchronous solutions. Additionally, we show that for a certain range of parameter values, the singular almost-synchronous solution is stable. In Section 4, we 


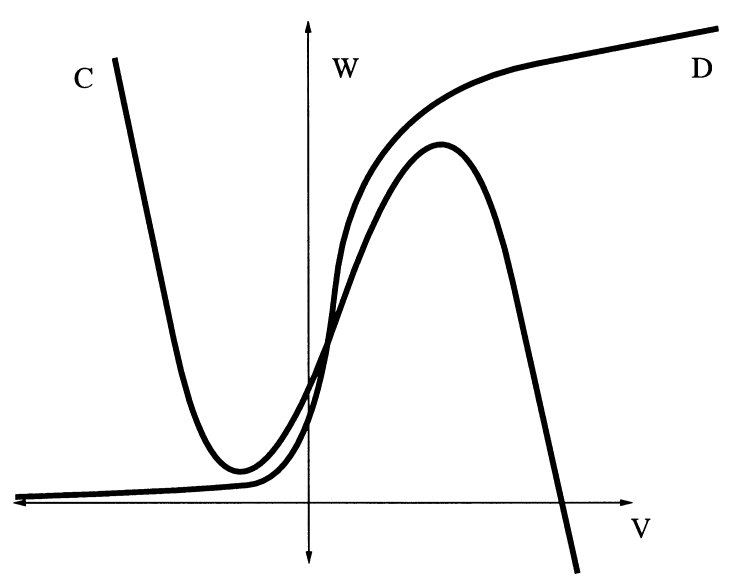

Fig. 1. The nullclines of (2.1).

show that the results concerning the existence and stability of singular solutions perturb to $\epsilon$ small. In Section 5 , we provide numerical simulations of our model equations. In this section, we also show how modulating the intrinsic and synaptic thresholds can produce stable synchronous solutions. Finally, in Section 6, we provide a discussion where we compare our work to some of the prior work in this area.

\section{Equations and statement of results}

\subsection{Equations}

We start with equations for a single oscillator, which already contain the three time scales. The equations are

$$
v^{\prime}=f(v, w), \quad w^{\prime}=\frac{\epsilon g(v, w)}{\tau_{\infty}(v)},
$$

where $\epsilon \ll 1$ is the singular perturbation parameter. The $v$-nullcline is the curve $C=\{(v, w): f(v, w)=0\}$ and is cubic shaped. The $w$-nullcline $D=\{(v, w): g(v, w)=0\}$ is a non-decreasing graph that intersects $C$ at exactly one point (see Fig. 1). The parameters will always be chosen so that $C$ and $D$ intersect along the middle branch of $C$, thus insuring that an isolated neuron is oscillatory. We assume that $f>0(<0)$ below (above) $C$ and $g>0(<0)$ below (above) $D$. The function $\tau_{\infty}(v)$ is given by

$$
\tau_{\infty}(v)= \begin{cases}1 & \text { if } v<v_{\theta} \\ \epsilon / \gamma & \text { if } v \geq v_{\theta}\end{cases}
$$

Here the parameter $v_{\theta}$ is the threshold for entering the active phase and $\gamma$ governs the rate of passage through the active phase. We assume that $v_{\theta}$ lies between the two knees of $C$. It will also be necessary to assume that $\partial g / \partial v>0$ and near the left and right branches of $C, \partial g / \partial w<0$ and $\partial f / \partial w<0$.

We now add coupling that represents fast excitatory synapses. The full equations are

$$
v_{i}^{\prime}=f\left(v_{i}, w_{i}\right)-g_{\mathrm{syn}} H\left(v_{j}-v_{\mathrm{st}}\right)\left(v_{i}-v_{\mathrm{syn}}\right), \quad w_{i}^{\prime}=\frac{\epsilon g\left(v_{i}, w_{i}\right)}{\tau_{\infty}\left(v_{i}\right)},
$$


where $j \neq i$. Here $g_{\mathrm{syn}}$ is the conductance of the synaptic current, $H\left(v_{j}-v_{\mathrm{st}}\right)$ is the Heaviside function and $v_{\mathrm{st}}$ is the synaptic threshold. $v_{\text {syn }}$ is chosen to be high so that the synaptic current is inward; that is, $v_{i}-v_{\text {syn }}<0$ along the singular solutions. The $v$-nullcline is the same as that of the uncoupled cell when $v_{j}<v_{\mathrm{st}}$. For $v_{j}>v_{\mathrm{st}}$, the effect of the coupling term is to raise the nullcline and change its shape; if $g_{\text {syn }}$ is not too large, then the excited $v$-nullcline is still qualitatively cubic. We denote this cubic by $C_{1}$.

Note that there are two thresholds $v_{\theta}$ and $v_{\mathrm{st}}$ in the equations. For clarity, we assume, for now, that

$$
v_{\mathrm{st}}=v_{\theta}
$$

In the numerics section, we discuss how the results may change if this does not hold.

\subsection{Singular solutions}

All of the solutions are analyzed by treating $\epsilon$ as a small, singular perturbation parameter. We construct singular solutions after formally setting $\epsilon=0$; it is then necessary to prove that the singular solution perturbs for $\epsilon>0$ to an actual solution of the full equations. One step in constructing the singular solution is to use fast and slow time scales to derive equations for the fast and slow flows.

We first demonstrate how to construct the singular trajectory for a single uncoupled cell. When $\epsilon$ is set equal to zero in (2.1), we obtain the equations for the fast flow

$$
\begin{aligned}
v^{\prime} & =f(v, w), & w^{\prime}=0, \quad \text { if } v<v_{\theta}, \\
v^{\prime} & =f(v, w), & w^{\prime}=\gamma g(v, w), \quad \text { if } v \geq v_{\theta} .
\end{aligned}
$$

By introducing the rescaling $\tau=\epsilon t$ into (2.1) and then setting $\epsilon=0$, we obtain equations for the slow flow in the silent phase,

$$
0=f(v, w), \quad \dot{w}=g\left(v_{\mathrm{L}}(w), w\right), \quad \text { if } v<v_{\theta} .
$$

Differentiation is with respect to $\tau$ and $v_{\mathrm{L}}(w)$ is obtained by solving $0=f(v, w)$ along the left branch of the cubic C.

Note that Eqs. (2.3) and (2.5) are simply scalar equations; in (2.3), the variable $w$ serves as a parameter in the $v^{\prime}$ equation. The full two-dimensional system (2.1) has been reduced to two one-dimensional equations and solutions of these are easy to characterize. However, (2.4) is not reduced. In other fast-slow systems of the form (2.1), typically the entire $v$-nullcline consists of rest points for the fast flow. In our case, however, only the portion of $C$ with $v<v_{\theta}$ consists of rest points. Thus, the structure of these flows is quite different depending on whether cells are silent or active.

The singular periodic orbit for an uncoupled cell is shown in Fig. 2. The orbit begins at the left knee of $C$, which we denote by $\left(v_{\mathrm{LK}}, w_{\mathrm{LK}}\right)$. The first part of the singular orbit is a solution of (2.3) that connects $\left(v_{\mathrm{LK}}, w_{\mathrm{LK}}\right)$ to $\left(v_{\theta}, w_{\mathrm{LK}}\right)$. The second part is a solution of (2.4) that connects $\left(v_{\theta}, w_{\mathrm{LK}}\right)$ to $\left(v_{\theta}, w_{\mathrm{h}}\right)$, where $w_{\mathrm{h}}>w_{\mathrm{LK}}$; this corresponds to the active phase. The third part is a solution to (2.3) that connects $\left(v_{\theta}, w_{\mathrm{h}}\right)$ back to $C$ at some point $\left(v_{\mathrm{L}}\left(w_{\mathrm{h}}\right), w_{\mathrm{h}}\right)$. The fourth and final part is a solution to (2.5) that connects $\left(v_{\mathrm{L}}\left(w_{\mathrm{h}}\right), w_{\mathrm{h}}\right)$ to $\left(v_{\mathrm{LK}}, w_{\mathrm{LK}}\right)$.

The construction of a synchronous solution for the coupled system is done in the same manner as that of the periodic solution for the uncoupled cell; the only difference is that the dynamics are changed once the voltage passes across $v_{\mathrm{st}}=v_{\theta}$. Thus, if each $v_{i}>v_{\mathrm{st}}$, then (2.4) is replaced by

$$
v^{\prime}=f(v, w)-g_{\text {syn }}\left(v-v_{\text {syn }}\right), \quad w^{\prime}=\gamma g(v, w) .
$$




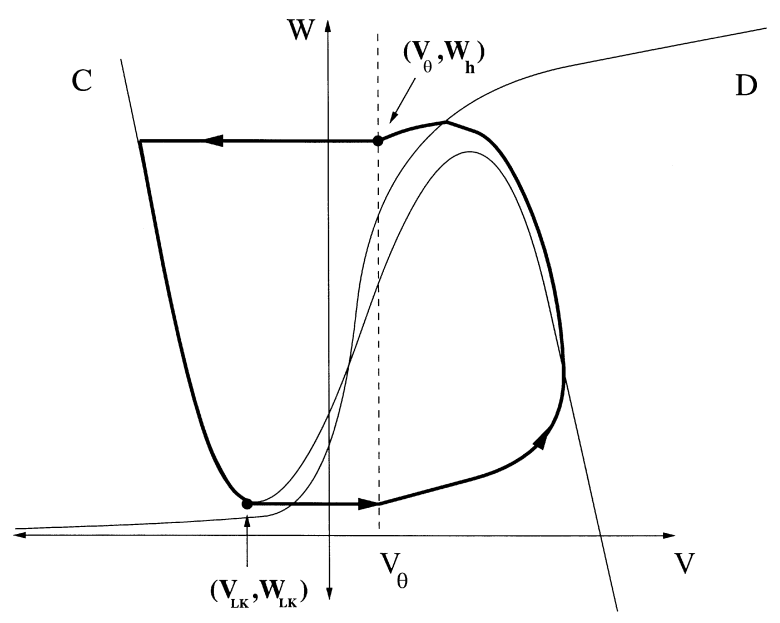

Fig. 2. The singular periodic orbit.

Note that the synchronous solution is not the same as the uncoupled periodic solution (see Fig. 3); this is because they satisfy different equations while in the active phase.

There is no difficulty in proving that these singular periodic solutions perturb, for $\epsilon>0$ small, to actual solutions of either (2.1) or (2.2) with $v_{1} \equiv v_{2}$ and $w_{1} \equiv w_{2}$. See, for example, [10]. The actual periodic orbits for $\epsilon$ small lie $\mathrm{O}(\epsilon)$ close to the singular orbits in phase space, except near the left knee of $C$, where the solution is typically $\mathrm{O}\left(\epsilon^{a}\right), a>0$, close to the singular solution. Note that the duration of each of these solutions in the silent phase is $\mathrm{O}(1)$ in $\tau$-time, i.e. $\mathrm{O}(1 / \epsilon)$ in $t$-time. By contrast, the $t$-time in the active phase is $\mathrm{O}(1 / \gamma)$. Hence, if $\epsilon \ll \gamma$, then the active phase is very short compared to the silent phase.

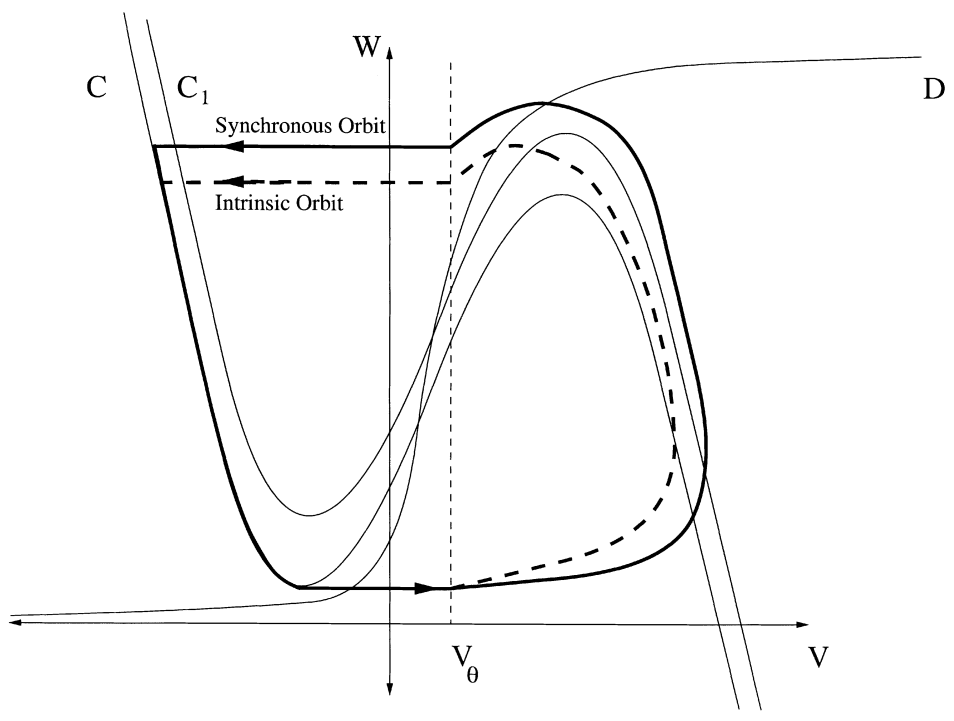

Fig. 3. The synchronous and intrinsic orbits. 


\subsection{Almost-synchronous solutions}

When we say that a solution is almost-synchronous, or an $\mathrm{O}(\epsilon)$-synchronous solution, we shall mean that the cells cross the threshold $v_{\theta}$ at time differences that are small compared to the period of the oscillation. In phase space, however, the solution will be far from synchronous in the sense that the values of the voltage and recovery variables at which the cells jump to the activated state have a finite difference that does not go to zero as the small parameter $\epsilon$ goes to zero.

A non-synchronous singular solution is constructed as before, composed of pieces to fast and slow equations. As before, if both cells are silent (i.e. $v<v_{\theta}$ ) the fast flow is governed by (2.3) and the slow flow by (2.5). If both cells are active the fast flow for each cell is governed by (2.6) and there is no slow flow. Since the voltages of the two cells may not be identical, it is possible that one of the cells is silent while the other is active. In that case, the active cell is governed by Eq. (2.4) while the silent one is governed by

$$
v^{\prime}=f(v, w)-g_{\mathrm{syn}}\left(v-v_{\mathrm{syn}}\right), \quad w^{\prime}=0 .
$$

The singular version of the almost-synchronous periodic solution is constructed as a fixed point of a Poincare map: cell 1 starts at the left knee of $C$ and cell 2 at some point on the left branch of $C$ (and $\mathrm{O}(\gamma)$ in phase space) above the knee. The analysis follows the points until one of the cells again reaches the original knee. If the other is at the original starting point of cell 2, we have a fixed point for the Poincare map, corresponding to a periodic singular solution. To show that this corresponds to an almost-synchronous solution, we provide estimates to show that the times at which the cells cross $v_{\theta}$ differ by an amount that is an $\mathrm{O}(\epsilon)$ fraction of the period.

We now describe the singular trajectory more explicitly; as before, it consists of many pieces. The construction holds if the cell not initially at the knee of $C$ starts low enough to be below the left knee of $C_{1}$. The first portion begins as cell 1 leaves the left knee. In this portion, cell 1 is governed by $(2.3)$ and has a trajectory in $(v, w)$ space that is horizontal until it crosses $v_{\theta}$ at $t=t_{1}$. During the time $t<t_{1}$, cell 2 is governed by (2.3) in which $w^{\prime}=0$, so cell 2 does not move.

At time $t_{1}, H\left(v_{1}-v_{\theta}\right)$ switches from 0 to 1 , so cell 2 is then governed by (2.7), while cell 1 is governed by (2.4). Since, by hypothesis, cell 2 is below the left knee of $C_{1}$, cell 2 can now move, and does so horizontally until it reaches $v_{\theta}$ at $t_{2}$. Note that for $t_{1}<t<t_{2}$, cell 1 moves both horizontally and vertically, because it is governed by (2.4) (see Fig. 4, we assume, for now, that $\left.v_{1}\left(t_{2}\right)>v_{\theta}\right)$. At time $t_{2}, H\left(v_{2}-v_{\theta}\right)$ switches from 0 to 1 . Hence cell 1 is then governed by (2.6). Furthermore, since cell 2 is also being excited and $v_{1}>v_{\theta}$, cell 2 is also governed by (2.6). Following (2.6), both cells eventually reach $v_{\theta}$ again. (In Fig. 4, this part of the trajectory is schematic; later figures take into account more of the structure of the equations and different cases.)

Assume that cell 1 is the first to cross $v_{\theta}$ again, at $t=T_{1}$. After this time, cell 2 satisfies (2.4) until it crosses $v_{\theta}$ at $T_{2}$. For $T_{1}<t<T_{2}$, cell 1 is governed by (2.7). Once a cell crosses $v_{\theta}$, it moves horizontally towards a point on the left branch of $C$. The cells then evolve along the left branch of $C$ until one of the cells reaches the left knee of $C$. A similar scenario holds if cell 2 is the first to reach $v_{\theta}$ from the active branch.

Much of the analysis concerns the parts of the trajectories in phase space in which the two cells are governed by different equations. In terms of voltages, let $v_{\mathrm{F}}$ denote the $v$-value of the trajectory of cell 1 at $t=t_{2}$ (see Fig. 5). Then for $v_{\theta}<v<v_{\mathrm{F}}$ the two cells satisfy different equations. Over this range of voltages, cell 1 does not receive excitation from cell 2, and hence satisfies (2.4). Cell 2 does however receive excitation when it is in that portion of the phase plane, so it is governed by (2.6). As we show below, this difference causes a separation between the orbits of the cells which is bounded from below by $k_{1} \gamma$ and from above by $k_{2} \gamma$ for some $k_{2}>k_{1}>0$, independent of the initial difference, providing the latter is small enough. Beyond $v_{\mathrm{F}}$, the two cells satisfy the same equations until 


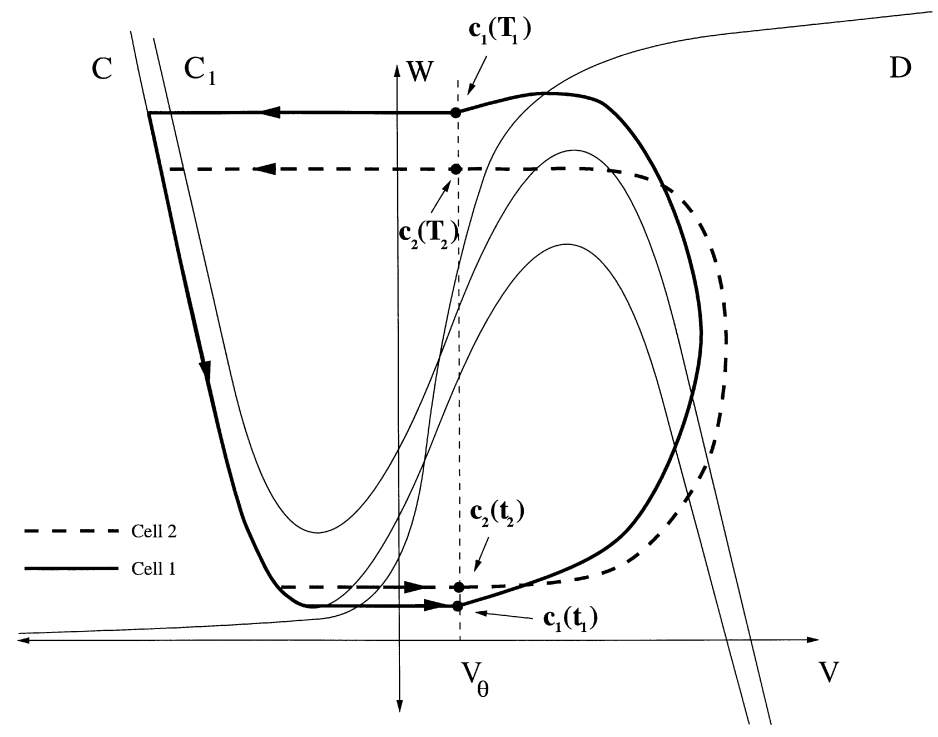

Fig. 4. A general phase plane for cells 1 and 2. The times $t_{1}<t_{2}<T_{1}<T_{2}$ denote the times a particular cell crosses the line $v=v_{\theta}$.

one of the cells again crosses $v_{\theta}$ on the jump down. A similar analysis on the jump down shows that the difference in governing equations leads to the maintenance of the $\mathrm{O}(\gamma)$ separation at the end of that jump.

The singular flow naturally gives rise to a one-dimensional map, which we denote by $\Pi$. More precisely, if $\left(v_{2}, w_{2}\right)$ is the initial position of cell 2 on the left branch of $C$ and $(\hat{v}, \hat{w})$ is the position of the trailing cell after the other cell has returned to the left knee, then $\Pi\left(w_{2}\right)=\hat{w}$. This map is well defined if $w_{2}-w_{\text {LK }}$ is sufficiently

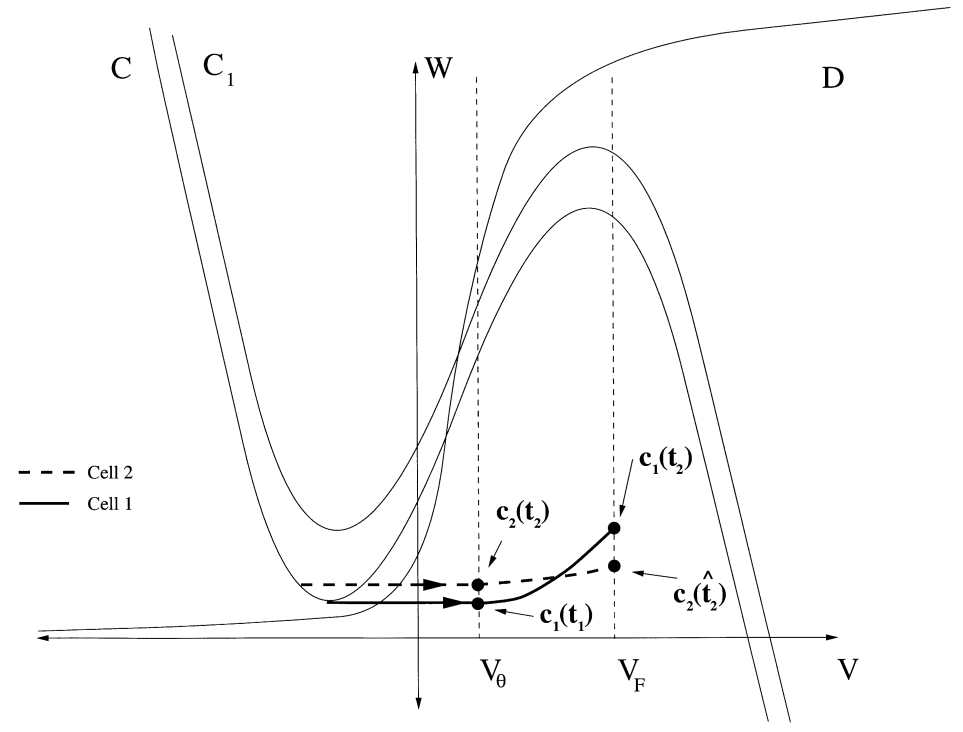

Fig. 5. The expansion in the up jump over the $v$-range $\left[v_{\theta}, v_{\mathrm{F}}\right]$. The ordering of times is $t_{1}<t_{2}<\hat{t}_{2}$. Cell 1 is governed by (2.4) and cell 2 is governed by (2.6) over this range of $v$-values. 


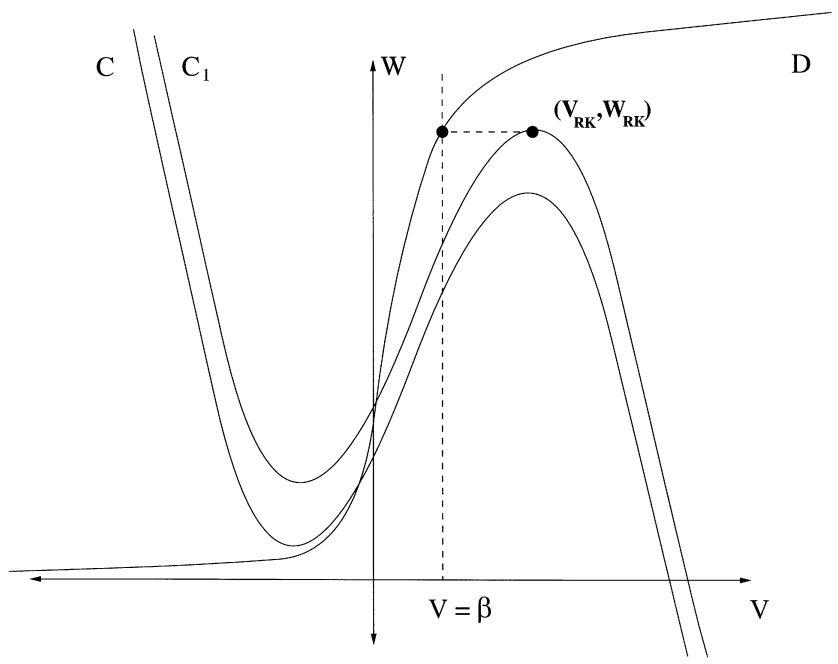

Fig. 6. The graphical description of $v=\beta$. The value $\beta$ is obtained by solving $g\left(\beta, w_{\mathrm{LK}}\right)=0$, where $w_{\mathrm{LK}}$ is the $w$-value of the left knee of the excited cubic $C_{1}$.

small; in particular, cell 2 should lie below the left knee of the excited cubic $C_{1}$. A fixed point of $\Pi$ corresponds to a periodic singular solution. The orbit may be orientation preserving or orientation reversing, depending on whether the first cell to return to the left knee of $C$ is cell 1 or cell 2, respectively.

From this explicit description of the Poincare map, we can see why a fixed point corresponds to an $O(\epsilon)$ synchronous solution. Though the cells begin a cycle with their recovery variable $w$ at values that are $\mathrm{O}(\gamma)$ apart, they reach $v_{\theta}$ at times $t_{1}$ and $t_{2}$, respectively, that are $\mathrm{O}(1)$ apart in $t$; as we will see, this continues to be true for the full equations (2.2) in which $\epsilon>0$. Since the total period of the trajectory is $O(1 / \epsilon)$ in $t$-time, the time lag has a fraction $\mathrm{O}(\epsilon)$ of the total period.

In the following section, we give conditions guaranteeing that $\Pi$ defines a uniform contraction on some interval of $C$ that does not contain the left knee. The bounds on that interval give information about the degree of synchronicity of the periodic orbit associated with the fixed point of $\Pi$. The construction of the interval also gives information about what properties of the cells determine how far from synchronous is the singular periodic trajectory. We also prove that the latter perturbs, for $\epsilon$ small, to a stable periodic solution of (2.2).

\subsection{Main result}

We now state two theorems. The first is concerned with the existence and stability of almost-synchronous singular solutions. Recall that these correspond to fixed points of the map П, defined in the previous section. As described above, the map П may be orientation preserving or reversing near the fixed point we seek; thus Theorem 1 has two sets of hypotheses, corresponding to these cases. Theorem 2 demonstrates that the almost-synchronous singular solutions perturb for small, positive $\epsilon$ to $\mathrm{O}(\epsilon)$-synchronous solutions of (2.2).

Before stating the theorems, it will be necessary to introduce some notation. Assume that the right knee of $C_{1}$ is at $\left(v_{\mathrm{RK}}, w_{\mathrm{RK}}\right)$ and choose $\beta$ so that $g\left(\beta, w_{\mathrm{RK}}\right)=0$ (see Fig. 6$)$.

Definition 1. The $\gamma$-dependent constants $\delta_{1}$ and $\delta_{2}$ are said to be $\tilde{O}(\gamma)$-apart if there exist $K_{1}$ and $K_{2}$ such that $K_{1} \gamma<\left|\delta_{1}-\delta_{2}\right|<K_{2} \gamma$ for $\gamma$ small. 
Note that the phrase $\tilde{\mathrm{O}}(\gamma)$-apart implies a two sided bound (lower and upper) on the quantities in question; alternatively, the phrase $\mathrm{O}(\gamma)$ implies simply an upper bound on a given quantity. In what follows, $C_{i}, K_{i}$ and $M_{i}, i=0,1,2, \ldots$, will be positive constants that do not depend on $\epsilon$ or $\gamma$.

Theorem 1. Suppose that the constants $v_{\theta}, \beta$ and $g_{\text {syn }}$ satisfy one of the following conditions:

(R) Either $v_{\theta}>\beta$, or $v_{\theta}<\beta$ and $\beta-v_{\theta}$ is sufficiently small compared to $g_{\mathrm{syn}}$;

(P) $v_{\theta}<\beta$ and $g_{\text {syn }}$ is sufficiently small compared to $\beta-v_{\theta}$.

Then for $\gamma$ sufficiently small, there exist $\Delta_{\exp }<\Delta_{\text {com }}$ such that $\Pi$ has a unique asymptotically stable fixed point $w_{*}$ on $\left(\Delta_{\exp }, \Delta_{\text {com }}\right)$. The distance between $w_{*}$ and $w_{\mathrm{LK}}$ is $\tilde{\mathrm{O}}(\gamma)$-apart. If $(\mathrm{P})$ is satisfied then $\Pi$ is orientation preserving on $\left(\Delta_{\exp }, \Delta_{\mathrm{com}}\right)$, while if $(\mathrm{R})$ is satisfied then $\Pi$ is orientation reversing on $\left(\Delta_{\exp }, \Delta_{\mathrm{com}}\right)$.

Remark 1. We will show that over the interval $v_{\theta}<v<v_{\mathrm{F}}$, the orbits of the two cells that start below some minimum distance $\left(\Delta_{\exp }\right)$ from each other separate to create a difference of size $\tilde{\mathrm{O}}(\gamma)$ independent of initial conditions. The order of magnitude of this distance is then shown to be maintained in the rest of the cycle. The conditions in (R) or $(\mathrm{P})$ are needed to maintain that distance. The parameter regimes in which both those conditions are violated correspond to the reconvergence of the separated trajectories during the down jump part of the trajectory. Case (R) is demonstrated for $v_{\theta}>\beta$; the conclusion of Theorem 1 holds for $v_{\theta}<\beta$, with $\beta-v_{\theta}$ small enough, by continuous dependence on initial conditions. Note that the condition $v_{\theta}<\beta$ alone is not sufficient to determine whether or not the solution is order preserving or reversing. It is the relation of $g_{\mathrm{syn}}$ to $\beta-v_{\theta}$ which determines which case holds. In particular, see Remark 6 for estimates on the parameters $v_{\theta}, \beta$ and $g_{\mathrm{syn}}$ that insure that case $(\mathrm{P})$ holds when $v_{\theta}<\beta$. For some range of $v_{\theta}<\beta$, neither $(\mathrm{P})$ nor $(\mathrm{R})$ holds, and there may be solutions that are more synchronous than our $\mathrm{O}(\epsilon)$-synchronous solutions.

Theorem 2. The asymptotically stable singular periodic solution given by Theorem 1 perturbs, for $\epsilon>0$, to an asymptotically stable $\mathrm{O}(\epsilon)$-solution of (2.2).

\section{Proof of Theorem 1}

There are three parts to the proof. In the first, we show that there is a regime of initial conditions, with spatial differences in the initial conditions of size $\mathrm{O}(\gamma)$, that expands under $\Pi$. This is the part that depends critically on the intermediate time scale $\mathrm{O}(\gamma)$. The proof shows that the two cells satisfy different equations over some parts of the trajectory, and that this creates the expansion.

In the second part, we show that initial conditions that start far enough away, but still at a spatial distance of size $\mathrm{O}(\gamma)$, are brought closer together under $\Pi$. This part is more straightforward. The argument can be thought of as a regular perturbation of the "fast threshold modulation" description in [16]; it does not use the subtleties associated with the intermediate time scale.

These two estimates help to create an interval in initial conditions whose image under $\Pi$ has points on both sides of the diagonal in $(\Delta, \Pi(\Delta))$ space. The third part of the proof shows that, in such an interval, $\Pi$ is uniformly contracting. This implies that there is a unique fixed point $\Pi(\Delta)=\Delta$. The estimates will enable us to see that, for this fixed point, the times at which the two cells cross the threshold $v=v_{\theta}$ are Õ(1)-apart, and hence the fixed point corresponds to an $\mathrm{O}(\epsilon)$-synchronous solution.

\subsection{Expansion}

We now assume, without loss of generality, that $w_{\mathrm{LK}}=0$. In this section, we prove that if $\Delta>0$ is sufficiently small, then $\Pi(\Delta)$ is not only greater than $\Delta$, but is bounded from below by some constant that does not depend on $\Delta$. How small $\Delta$ must be for that to hold does depend on $\gamma$, however. 
Proposition 3.1. There exist $C_{0}<C_{1}<C_{2}$ such that if $\Delta<C_{0} \gamma$ and $\gamma$ is sufficiently small, then $C_{1} \gamma<\Pi(\Delta)<$ $C_{2} \gamma$.

This result is proved by carefully following the singular orbit around in phase space as in the definition of $\Pi$. As before, we assume that cell 1 begins at the left knee of $C$ and cell 2 initially lies on the left branch of $C$ with $w_{2}(0)=\Delta$. Let $t_{1}, t_{2}, T_{1}$, and $T_{2}$ be as in Section 2.3 (see also Fig. 4).

The trajectory of each cell is horizontal until the cell crosses $v_{\theta}$. Hence, $w_{2}\left(t_{2}\right)-w_{1}\left(t_{1}\right)=\Delta$. The next lemma accounts for the primary source of expansion between the cells. This occurs when they first enter the active phase and satisfy different equations. As before, let $v_{\mathrm{F}}=v_{1}\left(t_{2}\right)$; we choose $\hat{t}_{2}$ so that $v_{2}\left(\hat{t}_{2}\right)=v_{\mathrm{F}}$ (see Fig 5).

Lemma 3.1. There exists $K_{0}$ such that if $\Delta<K_{0} \gamma$ and $\gamma$ is sufficiently small, then $w_{1}\left(t_{2}\right)$ and $w_{2}\left(\hat{t}_{2}\right)$ are $\tilde{\mathrm{O}}(\gamma)$-apart.

Proof. Since each $v_{i}(t)$ is increasing for $0 \leq t \leq \hat{t}_{2}$, we may consider the $w_{i}$ as functions of $v_{i}$. In this case, we write $W_{i}\left(v_{i}\right)$; that is, $W_{i}\left(v_{i}(t)\right)=w_{i}(t)$. Let

$$
\Phi(v, w, s)=\frac{g(v, w)}{f(v, w)-g_{\mathrm{syn}} s\left(v-v_{\mathrm{syn}}\right)} .
$$

Then for each $v \in\left[v_{\theta}, v_{\mathrm{F}}\right]$,

$$
W_{1}(v)=\gamma \int_{v_{\theta}}^{v} \Phi\left(\eta, W_{1}(\eta), 0\right) \mathrm{d} \eta, \quad W_{2}(v)=\Delta+\gamma \int_{v_{\theta}}^{v} \Phi\left(\eta, W_{2}(\eta), 1\right) \mathrm{d} \eta .
$$

Hence,

$$
W_{1}(v)-W_{2}(v)=-\Delta+\gamma \int_{v_{\theta}}^{v} \Phi\left(\eta, W_{1}(\eta), 0\right)-\Phi\left(\eta, W_{2}(\eta), 1\right) \mathrm{d} \eta .
$$

Choose $m_{1}, M_{1}$ and $M_{2}$ so that for $v_{\theta}<v<v_{\mathrm{F}}$ and $0 \leq s \leq 1$,

$$
-m_{1}<\frac{\partial}{\partial s} \Phi\left(v, W_{i}(v), s\right)<-M_{1}, \quad \text { and } \quad\left|\frac{\partial}{\partial w} \Phi\left(v, W_{i}(v), 0\right)\right|<M_{2} .
$$

The existence of $m_{1}$ and $M_{1}$ follows from our assumption that the synapse is excitatory. Let $M=v_{\mathrm{F}}-v_{\theta}$, $K_{0}=K_{1}=M M_{1} / 4$ and $K_{2}=3 M m_{1}$. Assume that $\Delta<K_{0} \gamma$. Then, from (3.1),

$$
\begin{aligned}
W_{1}(v)-W_{2}(v) & <\Delta+\gamma \int_{v_{\theta}}^{v} \frac{\partial \Phi}{\partial s}(-1)+\left|\frac{\partial \Phi}{\partial w}\right|\left|W_{1}-W_{2}\right| \mathrm{d} \eta \\
& <\Delta+\gamma m_{1} M+\gamma M_{2} \int_{v_{\theta}}^{v}\left|W_{1}(\eta)-W_{2}(\eta)\right| \mathrm{d} \eta
\end{aligned}
$$

We now apply Gronwall's inequality to obtain the upper bound:

$$
W_{1}(v)-W_{2}(v) \leq\left(\Delta+\gamma m_{1} M\right) \mathrm{e}^{\gamma M_{2} M}<2\left(\Delta+\gamma m_{1} M\right)<K_{2} \gamma,
$$

for $\gamma$ sufficiently small. In particular, if $v=v_{\mathrm{F}}$, then

$$
w_{1}\left(t_{2}\right)-w_{2}\left(\hat{t}_{2}\right)<K_{2} \gamma
$$

for $\gamma$ sufficiently small. 
In order to obtain a lower bound on $w_{1}\left(t_{2}\right)-w_{2}\left(\hat{t}_{2}\right)$, note that for $v_{\theta}<\eta<v_{\mathrm{F}}$,

$$
\begin{aligned}
\Phi & \left(\eta, W_{1}(\eta), 0\right)-\Phi\left(\eta, W_{2}(\eta), 1\right) \\
& =\left[\Phi\left(\eta, W_{2}(\eta), 0\right)-\Phi\left(\eta, W_{2}(\eta), 1\right)\right]+\left[\Phi\left(\eta, W_{1}(\eta), 0\right)-\Phi\left(\eta, W_{2}(\eta), 0\right)\right] \\
& \geq M_{1}-M_{2}\left|W_{1}(\eta)-W_{2}(\eta)\right| \geq M_{1}-M_{2} K_{2} \gamma \\
& >\frac{M_{1}}{2}
\end{aligned}
$$

if $\gamma$ is sufficiently small. It now follows from (3.1) with $v=v_{\mathrm{F}}$ that

$$
\begin{aligned}
w_{1}\left(t_{2}\right)-w_{2}\left(\hat{t}_{2}\right) & >-\Delta+\frac{\gamma M M_{1}}{2} \\
& >-K_{0} \gamma+\frac{\gamma M M_{1}}{2} \\
& >K_{1} \gamma .
\end{aligned}
$$

Remark 2. Note that the right-hand side of the last inequality for the lower bound $K_{1} \gamma$ has terms of both signs. This comes from the fact that the trajectories of the two cells, which satisfy different equations, cross paths between $v_{\theta}$ and $v_{\mathrm{F}}$. The negative term comes from the size of the initial difference in the $w$-components of the cells, and the positive term from the difference in equations that govern the cells. For small $K_{0}$, the sum is dominated by the latter, so the final distance is bounded below, independent of the initial distance.

Remark 3. During this initial excursion through the active phase, cell 2 feels coupling, but cell 1 does not. The effect of the excitatory coupling is to decrease the slope of the vector field of cell 2. This is the geometric reason why the cells enter the active phase with $w_{1}<w_{2}$ but when they cross $v=v_{\mathrm{F}}, w_{1}>w_{2}$ and expansion has taken place.

Remark 4. The estimates in Lemma 3.1 show that $w_{1}\left(t_{2}\right)$ and $w_{2}\left(\hat{t}_{2}\right)$ are also $\tilde{\mathrm{O}}\left(g_{\mathrm{syn}}\right)$-apart. This follows since both $K_{1}$ and $K_{2}$ contain either the factor $M_{1}$ or $m_{1}$, both of which are $\mathrm{O}\left(g_{\text {syn }}\right)$. Thus by making $g_{\text {syn }}$ sufficiently small, we can make the difference in the $w$-values of the cells arbitrarily small, independent of $\gamma$. This will be important for the expansion argument for the orientation preserving case. See also Remark 6.

We now follow each of the cells until they leave the active phase at $v=v_{\theta}$. These times are denoted as $T_{i}$. The following lemma implies that the $w$-components continue to be $\tilde{\mathrm{O}}(\gamma)$-apart through this part of the trajectory.

Lemma 3.2. If the conclusion of Lemma 3.1 is satisfied, then $w_{1}\left(T_{1}\right)$ and $w_{2}\left(T_{2}\right)$ are $\tilde{\mathrm{O}}(\gamma)$-apart (see Fig. 4).

Proof. We need to apply results from Fenichel's geometric singular perturbation theory [6]. Before applying this theory, we briefly summarize the results which are needed.

For small $\gamma$, there is a center manifold $R_{\gamma}$ for (2.6) that is $\mathrm{O}(\gamma)$ close to the right branch $R_{0}$ of the cubic $C_{1}$. In a neighborhood of $R_{\gamma}$, there is a Fenichel fibration. This associates to each $p_{0} \in R_{\gamma}$ a smooth, one-dimensional fiber; two points lie on the same fiber if they have the same 'asymptotic phase'. By definition, this means the following: suppose that $q_{1}$ and $q_{2}$ are two points which lie near $R_{\gamma}$ and let $q_{i}(t)$ be the solutions of (2.6) which pass through the $q_{i}$ at the same time, say $T_{0}$. Then $q_{1}$ and $q_{2}$ have the same asymptotic phase if there exist constants $B$ and $K$, which do not depend on the points or $\gamma$, such that

$$
\left\|q_{1}(t)-q_{2}(t)\right\|<B \mathrm{e}^{-K\left(t-T_{0}\right) / \gamma}
$$

for $\gamma$ sufficiently small and $t>T_{0}$, as long as these trajectories lie near $R_{\gamma}$. We note that the fibers do not correspond to actual solutions of (2.6). We will need the fact that the fibers depend smoothly on both $\gamma$ and the points $p_{0} \in R_{\gamma}$. 


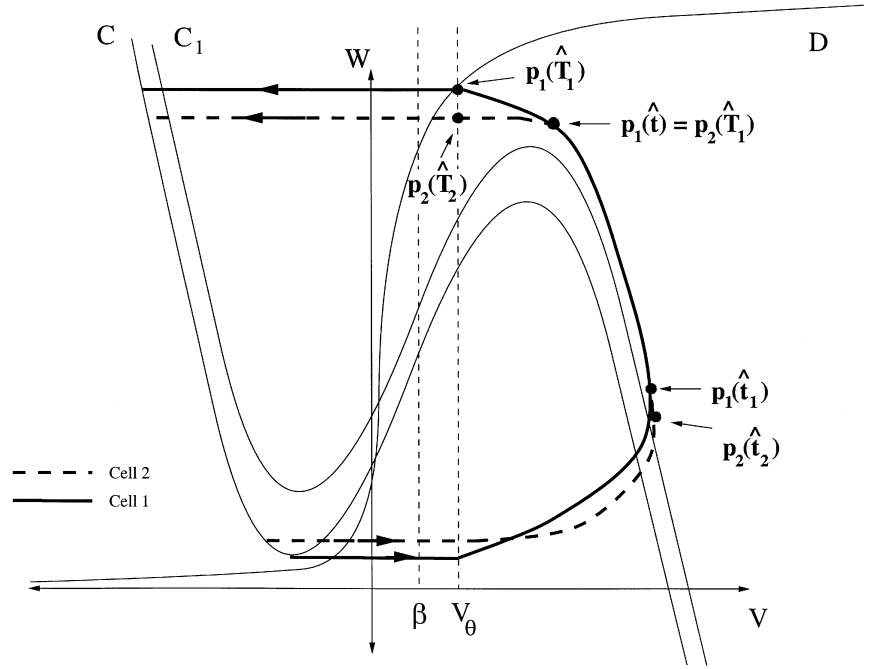

Fig. 7. The expansion in the down jump for case (R). Note that $p_{1}(t)$ and $p_{2}(t)$ have the same asymptotic phase as cell 1 and 2 , respectively. The ordering of times is $\hat{T}_{1}<\hat{T}_{2}$. The line $v=v_{\theta}$ lies to the right of $v=\beta$. Over the $v$-range $\left[\hat{v}_{2}\left(\hat{T}_{1}\right), v_{\theta}\right], p_{1}$ is governed by (2.6) while $p_{2}$ is governed by (2.4).

Now recall that cell 1 reaches $v=v_{\mathrm{F}}$ (at low values of $w$ ) with $t=t_{2}$ and cell 2 reaches that line with $t=\hat{t}_{2}$. For uniformity of notation, let $\hat{t}_{1} \equiv t_{2}$. Although $v_{\mathrm{F}}$ is not necessarily near $R_{\gamma}$, we can still determine the asymptotic phase of points which lie on $v_{\mathrm{F}}$ since the vector field forces all these points to eventually become near $R_{\gamma}$. Thus choose $p_{i} \in R_{\gamma}$ so that each point $\left(v_{\mathrm{F}}, w_{i}\left(\hat{t}_{i}\right)\right), i=1,2$, lies on the fiber associated to point $p_{i}$. Hence, if $p_{i}(t)$ is the solution of (2.6) that passes through $p_{i}$ at time $t=\hat{t}_{i}$, then

$$
\left\|\left(v_{i}(t), w_{i}(t)\right)-p_{i}(t)\right\|<B \mathrm{e}^{-K\left(t-\hat{t}_{i}\right) / \gamma},
$$

for $t>\hat{t}_{i}$ as long as the trajectories lie near $R_{\gamma}$. In particular, the trajectories $\left(v_{i}(t), w_{i}(t)\right)$ and $p_{i}(t)$ are exponentially close to each other when they eventually leave a small neighborhood of $R_{\gamma}$. This is shown in Fig. 7, which is a more accurate version of Fig. 4 for this part of the trajectory. It follows that if we now view $\left(p_{1}(t), p_{2}(t)\right)$ as a singular $(\epsilon=0)$ solution of the full system (2.2), then the places where cell $i$ and $p_{i}(t)$ leave the active phase are exponentially close to each other. Suppose that $p_{i}(t)=\left(\hat{v}_{i}(t), \hat{w}_{i}(t)\right)$ and choose $\hat{T}_{i}$ so that $\hat{v}_{i}\left(\hat{T}_{i}\right)=v_{\theta}$. Lemma 3.2 follows if we can show that $\hat{w}_{i}\left(\hat{T}_{i}\right)$ are $\tilde{O}(\gamma)$-apart.

We note that the $\hat{w}_{i}\left(\hat{t}_{i}\right)$ are $\tilde{\mathrm{O}}(\gamma)$-apart. This is true because the fibers through the $p_{i}$ are horizontal when $\gamma=0$, and the fibers depend smoothly on $\gamma$. Since, according to Lemma 3.1, the $w_{i}\left(\hat{t}_{i}\right)$ are $\tilde{O}(\gamma)$-apart, and each $\left(v_{\mathrm{F}}, w_{i}\left(\hat{t}_{i}\right)\right)$ and $p_{i}$ lie on the same fiber, we conclude that $\hat{w}_{i}\left(\hat{t}_{i}\right)$ are $\tilde{\mathrm{O}}(\gamma)$-apart. We further note that $\hat{w}_{1}\left(\hat{t}_{2}\right)$ and $\hat{w}_{2}\left(\hat{t}_{2}\right)$ are $\tilde{\mathrm{O}}(\gamma)$-apart. This is because

$$
\hat{w}_{1}\left(\hat{t}_{2}\right)-\hat{w}_{2}\left(\hat{t}_{2}\right)=\left(\hat{w}_{1}\left(\hat{t}_{2}\right)-\hat{w}_{1}\left(\hat{t}_{1}\right)\right)-\left(\hat{w}_{1}\left(\hat{t}_{1}\right)-\hat{w}_{2}\left(\hat{t}_{2}\right)\right) .
$$

We have just shown that the second term on the right-hand side is $\tilde{\mathrm{O}}(\gamma)$. The first term is positive because $\hat{t}_{2}>\hat{t}_{1}$; it is $\mathrm{O}(\gamma)$ because $\hat{t}_{2}-\hat{t}_{1}=\mathrm{O}(1)$ and $\hat{w}_{1}^{\prime}=\mathrm{O}(\gamma)$ on $R_{\gamma}$. It then follows that $\hat{w}_{1}\left(\hat{t}_{2}\right)$ and $\hat{w}_{2}\left(\hat{t}_{2}\right)$ are $\tilde{\mathrm{O}}(\gamma)$-apart.

For $\hat{t}_{i}<t<\hat{T}_{1}, p_{i}$ lie on the same one-dimensional curve that contains $R_{\gamma}$. It follows that $\hat{T}_{1}<\hat{T}_{2}$ and we can choose $\hat{t}<\hat{T}_{1}$ so that

$$
p_{1}(\hat{t})=p_{2}\left(\hat{T}_{1}\right) \equiv\left(\hat{v}_{H}, \hat{w}_{H}\right),
$$


as shown in Fig. 7. We claim that

$$
\hat{v}_{H}-v_{\theta}>K_{3}
$$

for some constant $K_{3}$ that does not depend on $\gamma$. To prove this, we first estimate $\hat{T}_{1}-\hat{t}$. Recall that for $\hat{t}_{i}<t<\hat{T}_{1}, p_{i}$ satisfy the same differential equations, but with different initial conditions. Hence, the time between these solutions remains invariant. In particular, if $\hat{t}_{0}$ is such that $p_{2}\left(\hat{t}_{2}+\hat{t}_{0}\right)=p_{1}\left(\hat{t}_{2}\right)$, then $\hat{T}_{1}-\hat{t}=\hat{t}_{0}$. Now, $\hat{w}_{2}^{\prime}=\mathrm{O}(\gamma)$ for $\hat{t}_{2}<t<\hat{t}_{2}+\hat{t}_{0}$. Since $\hat{w}_{1}\left(\hat{t}_{2}\right)$ and $\hat{w}_{2}\left(\hat{t}_{2}\right)$ are $\tilde{\mathrm{O}}(\gamma)$-apart, we conclude that $\hat{T}_{1}-\hat{t}=\hat{t}_{0}=\mathrm{O}(1)$ with respect to $\gamma$. Away from the cubics, $\hat{v}_{1}^{\prime}$ is bounded from zero by a constant that does not depend on $\gamma$. Since $\hat{v}_{H}-v_{\theta}=\hat{v}_{1}\left(\hat{T}_{1}\right)-\hat{v}_{1}(\hat{t})$ the inequality (3.2) now follows.

We now estimate $\left|\hat{w}_{1}\left(\hat{T}_{1}\right)-\hat{w}_{2}\left(\hat{T}_{2}\right)\right|$. To do this, we divide the proof into two cases given in the statement of Theorem 1. First suppose that $(\mathrm{R})$ is satisfied and $v_{\theta}>\beta$. In this case, each $\hat{w}_{i}(t)$ must increase until the $p_{i}(t)$ cross $v_{\theta}$, as shown in Fig. 7. This is because $v_{\theta}>\beta$ implies that for $v>v_{\theta}$, the trajectory is in the region in which $w^{\prime}>0$. For $\hat{t}_{i}<t<\hat{T}_{1}, p_{i}$ satisfy the same equations with different initial conditions that are $\tilde{\mathrm{O}}(\gamma)$-apart. In the rescaled time $\eta=\gamma t$, each $\hat{w}_{i}^{\prime}(\eta)$ is positive, bounded from above and bounded away from zero. It then follows that $\hat{w}_{1}\left(\hat{T}_{1}\right)$ and $\hat{w}_{2}\left(\hat{T}_{2}\right)$ remain $\tilde{\mathrm{O}}(\gamma)$-apart. In particular, there exist $K_{4}$ and $K_{5}$

$$
K_{4} \gamma<\hat{w}_{1}\left(\hat{T}_{1}\right)-\hat{w}_{2}\left(\hat{T}_{1}\right)<K_{5} \gamma
$$

Since $\hat{w}_{2}^{\prime}>0$ for $\hat{T}_{1}<T<\hat{T}_{2}$, we conclude that

$$
\hat{w}_{1}\left(\hat{T}_{1}\right)-\hat{w}_{2}\left(\hat{T}_{2}\right)<\hat{w}_{1}\left(\hat{T}_{1}\right)-\hat{w}_{2}\left(\hat{T}_{1}\right)<K_{5} \gamma
$$

We now establish a lower bound for $\hat{w}_{1}\left(\hat{T}_{1}\right)-\hat{w}_{2}\left(\hat{T}_{2}\right)$. At time $t=\hat{T}_{1}, p_{2}$ stops receiving excitation from $p_{1}$, thus causing a switch of the governing equation for $p_{2}$ for the remainder of its active phase. In particular, over the range $v_{\theta}<v<\hat{v}_{H}, p_{2}$ is governed by (2.4) whereas $p_{1}$ is governed by (2.6). This situation is analogous to Lemma 3.1. To better understand the present case, let $W_{i}(v)$ and $\Phi$ be as in the proof of Lemma 3.1 and choose $\hat{M}_{1}$ and $\hat{M}_{2}$ so that

$$
\frac{\partial}{\partial s} \Phi\left(v, W_{i}(v), s\right)<-\hat{M}_{1}, \quad \text { and } \quad\left|\frac{\partial}{\partial w} \Phi\left(v, W_{i}(v), 0\right)\right|<\hat{M}_{2} .
$$

Then integrating the governing equations,

$$
W_{1}\left(v_{\theta}\right)=\hat{w}_{H}+\gamma \int_{\hat{v}_{H}}^{v_{\theta}} \Phi\left(\eta, W_{1}(\eta), 1\right) \mathrm{d} \eta, \quad W_{2}\left(v_{\theta}\right)=\hat{w}_{H}+\gamma \int_{\hat{v}_{H}}^{v_{\theta}} \Phi\left(\eta, W_{2}(\eta), 0\right) \mathrm{d} \eta .
$$

Subtracting the two equations and reversing the limits of integration (since $v_{\theta}<\hat{v}_{H}$ ) yields,

$$
\begin{aligned}
\hat{w}_{1}\left(\hat{T}_{1}\right)-\hat{w}_{2}\left(\hat{T}_{2}\right) & =W_{1}\left(v_{\theta}\right)-W_{2}\left(v_{\theta}\right)=\gamma \int_{v_{\theta}}^{\hat{v}_{H}} \Phi\left(\eta, W_{2}(\eta), 0\right)-\Phi\left(\eta, W_{1}(\eta), 1\right) \mathrm{d} \eta \\
& =\gamma \int_{v_{\theta}}^{\hat{v}_{H}}-\frac{\partial}{\partial s} \Phi\left(\eta, W\left(\eta^{*}\right), s^{*}\right)+\frac{\partial}{\partial w} \Phi\left(\eta, W\left(\eta^{*}\right), 0\right)\left(W_{2}-W_{1}\right) \mathrm{d} \eta \\
& >\gamma\left(\hat{v}_{H}-v_{\theta}\right)\left(\hat{M}_{1}-\gamma K_{2} \hat{M}_{2}\right)>\frac{\gamma \hat{M}_{1} K_{3}}{2} .
\end{aligned}
$$

Together with (3.3), this demonstrates that $\hat{w}_{i}\left(\hat{T}_{i}\right)$ are $\tilde{\mathrm{O}}(\gamma)$-apart.

The above argument shows that Lemma 3.2 holds if $(\mathrm{R})$ is satisfied and $v_{\theta}>\beta$. By continuous dependence of solutions, Lemma 3.2 still holds if $(\mathrm{R})$ is satisfied with $v_{\theta}<\beta$ and $\beta-v_{\theta}$ is sufficiently small. Note that cell 2 returns to the left branch below cell 1 . Thus their orientations have been reversed. 


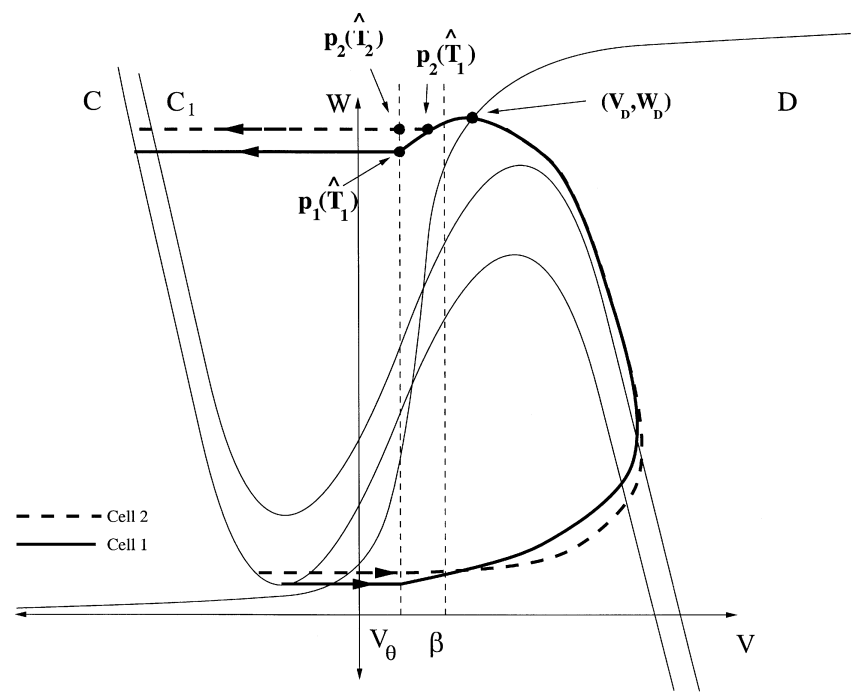

Fig. 8. The expansion in the down jump for case (P). The ordering of times is $\hat{T}_{1}<\hat{T}_{2}$. The line $v=v_{\theta}$ lies to the left of $v=\beta$. Over the $v$-range $\left[\hat{v}_{2}\left(\hat{T}_{1}\right), v_{\theta}\right], p_{1}$ is governed by (2.6) while $p_{2}$ is governed by (2.4).

Now suppose that $(\mathrm{P})$ is satisfied. In this case, let $p_{1}(t)$ cross the $w$-nullcline at $\left(v_{D}, w_{D}\right)$. Thus $\hat{w}_{1}(t)$ increases to a maximum value $w_{D}$ and then decreases. First assume that $\hat{w}_{2}\left(\hat{T}_{1}\right)$ lies above the $w$-nullcline $D$, as in Fig. 8 . As before, over the interval $\hat{v}_{2}\left(\hat{T}_{1}\right)$ to $v_{\theta}, p_{2}$ satisfies (2.4) while $p_{1}$ satisfied (2.6). As above, this creates an $\tilde{\mathrm{O}}(\gamma)$ difference in the $\hat{w}$ values. Thus, $\hat{w}_{2}\left(\hat{T}_{2}\right)$ and $\hat{w}_{1}\left(\hat{T}_{1}\right)$ differ by $\tilde{O}(\gamma)$, as desired.

It remains to show that the sufficient condition that $\hat{w}_{2}\left(\hat{T}_{1}\right)$ lies above $D$ can be satisfied. This is the case if the distance between $p_{1}$ and $p_{2}$ can be made sufficiently small. Recall from Remark 4 that we have an explicit upper bound on the amount of expansion of the $p_{i}$ in terms of the parameter $g_{\text {syn }}$. Thus, by making $g_{\text {syn }}$ sufficiently small, we can guarantee that $\hat{w}_{2}\left(\hat{T}_{2}\right)$ is $\mathrm{O}(\gamma)$ above $\hat{w}_{1}\left(\hat{T}_{1}\right)$. Finally, since cell 1 returns to the left branch below cell 2 , the orientation of the cells has been preserved. This completes the proof of Lemma 3.2.

Remark 5. Note that in the estimate for the lower bound in Lemma 3.1, there were two terms, one of which came from differences in initial conditions and the other from the difference in governing equations. In the down jump, there is only one term corresponding to the difference in governing equations (3.4), since the $p_{i}$ both pass through $\left(\hat{v}_{H}, \hat{w}_{H}\right)$. As discussed in Remark 3, this $\tilde{\mathrm{O}}(\gamma)$ difference occurs because the magnitude of the 'slope' of the uncoupled cell, $p_{2}$, is less than that of the coupled cell, $p_{1}$.

It is now a simple matter to complete the proof of Proposition 3.1. For $t>\hat{T}_{i}$, each cell moves horizontally to the left under the fast flow until it reaches the nullcline $C$. Each is then governed by (2.5) until one of the cells reaches $v=v_{\text {LK }}$. Over that range, the distance between the cells is compressed. However, the amount apart is bounded below, independent of initial conditions if the latter is sufficiently small. Thus, by choosing $C_{0}$ small enough, we can obtain the expansion in the statement of Proposition 3.1. The upper bound in that statement also follows.

Remark 6. The arguments in the proof of Lemma 3.2 provide a simple estimate for $g_{\mathrm{syn}}$ to satisfy condition (P). Let $p_{1}(t)$ cross the $w$-nullcline at $t=\hat{t}_{D}$. Let $\delta$ denote the time from $p_{2}\left(\hat{t}_{D}\right)$ to $p_{1}\left(\hat{t}_{D}\right)$ under the dynamics $(2.6)$. Let $\sigma=\hat{T}_{1}-\hat{t}_{D}$. The sufficient condition for $(\mathrm{P})$, recast in terms of these newly defined quantities, is $\delta<\sigma$. We now estimate $\delta$ and $\sigma$. 


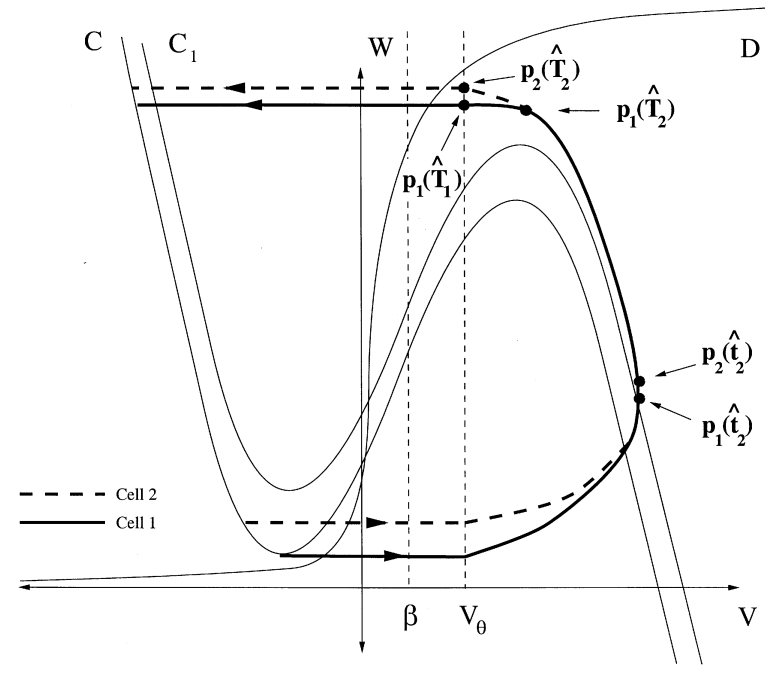

Fig. 9. Compression for case (R). The ordering of times is $\hat{T}_{2}<\hat{T}_{1}$. Over the $v$-range $\left[\hat{v}_{1}\left(\hat{T}_{2}\right), v_{\theta}\right], p_{2}$ is governed by $(2.6)$ while $p_{1}$ is governed by (2.4). Note for these initial conditions, the cells preserve their orientation.

Let $N=\max \left|f(v, w)-g_{\mathrm{syn}}\left(v-v_{\mathrm{syn}}\right)\right|$ for $v_{\theta}<v<v_{D}$ and $w>w_{\mathrm{RK}}$. Then

$$
\sigma \geq \frac{v_{D}-v_{\theta}}{N} \geq \frac{\beta-v_{\theta}}{N}
$$

Note that $\beta-v_{\theta}$ is independent of $g_{\text {syn }}$ and $N$ is $\mathrm{O}(1)$ with respect to $g_{\text {syn }}$. Next we estimate the time $\delta$. The time between the $p_{i}$ is invariant as these $p_{i}$ evolve along $R_{\gamma}$ to $v=v_{D}$. On $R_{\gamma}, \hat{w}_{i}^{\prime}$ is $\mathrm{O}(\gamma)$ while the distance between the $\hat{w}_{i}$ is $\mathrm{O}\left(g_{\text {syn }} \gamma\right)$, by Remark 4. Thus the time between the $\hat{w}_{i}$ is $\mathrm{O}\left(g_{\text {syn }}\right)$. Therefore the simple condition

$$
\frac{\beta-v_{\theta}}{N}>\mathrm{O}\left(g_{\text {syn }}\right)
$$

implies that the parameters fall into case $(\mathrm{P})$.

\subsection{Compression}

We again start with cell 1 at the left knee and cell 2 on the left branch with height $\Delta$ above the knee. It is also assumed that cell 2 lies below the left knee of $C_{1}$. We show that if $\Delta$ is sufficiently large (but still $\mathrm{O}(\gamma)$ ), then the cells return after one cycle with a closer distance. More precisely, we prove

Proposition 3.2. There exists $M_{0}$ such that if $\Delta>M_{0} \gamma$, then $\Pi(\Delta)<\Delta$.

Proof. As before, we estimate the distance between the cells as they traverse through different portions of phase space. We use much of the same notation as in the proof of Proposition 3.1. In particular, let $p_{i}=\left(\hat{v}_{i}, \hat{w}_{i}\right)$ be the point on the center manifold $R_{\gamma}$ which has the same asymptotic phase as cell $i$ at $t=\hat{t}_{i}$ (see Fig. 9). Analysis similar to that in Lemma 3.1 shows that there exists $M_{3}$ such that for each $i=1,2$,

$$
0<\hat{w}_{i}-w_{i}(0)<M_{3} \gamma
$$


Therefore, if $\Delta>M_{3} \gamma$, then

$$
0<\hat{w}_{2}-\hat{w}_{1}<\Delta+M_{3} \gamma
$$

There is an important difference between the argument in the expansion part and the current situation. In the former, with $\Delta$ small enough, the fact that the cells satisfy different equations in some voltage interval leads to the crossing of the trajectories and the expansion of the initial spatial differences. However, the $\tilde{\mathrm{O}}(\gamma)$ expansion is bounded, independent of $\Delta$, and for large enough $\Delta$ (but still $\mathrm{O}(\gamma)$ ), this effect is not enough to cause the crossing of trajectories. Thus, we have $p_{2}$ above $p_{1}$ on $R_{\gamma}$ unlike the estimates with small difference in initial conditions. Hence, $p_{2}(t)$ will leave the active phase before $p_{1}(t)$ does so; i.e. $\hat{T}_{2}<\hat{T}_{1}$.

We next follow $p_{1}(t)$ and $p_{2}(t)$ for $\hat{t}_{2}<t<\hat{T}_{2}$. It is during this time that compression takes place. Note that $\hat{t}_{1}<\hat{t}_{2}$. Hence, $\hat{w}_{1}\left(\hat{t}_{1}\right)<\hat{w}_{1}\left(\hat{t}_{2}\right)$ and

$$
\begin{aligned}
\hat{w}_{2}(t)-\hat{w}_{1}(t) & =\hat{w}_{2}\left(\hat{t}_{2}\right)-\hat{w}_{1}\left(\hat{t}_{2}\right)+\gamma \int_{\hat{t}_{2}}^{t} g\left(\hat{v}_{2}, \hat{w}_{2}\right)-g\left(\hat{v}_{1}, \hat{w}_{1}\right) \mathrm{d} \tau \\
& <\hat{w}_{2}-\hat{w}_{1}+\gamma \int_{\hat{t}_{2}}^{t} g\left(\hat{v}_{2}, \hat{w}_{2}\right)-g\left(\hat{v}_{1}, \hat{w}_{1}\right) \mathrm{d} \tau \\
& <\Delta+M_{3} \gamma+\gamma \int_{\hat{t}_{2}}^{t} \frac{\partial g}{\partial v}\left(\hat{v}_{2}-\hat{v}_{1}\right)+\frac{\partial g}{\partial w}\left(\hat{w}_{2}-\hat{w}_{1}\right) \mathrm{d} \tau .
\end{aligned}
$$

Recall that near $R_{\gamma}, \partial g / \partial v>0$ and $\partial g / \partial w<0$. Let $M_{4}=\min \{|\partial g / \partial w|\}$ near $R_{\gamma}$. Since the slope of $R_{\gamma}$ is negative, $\hat{v}_{2}-\hat{v}_{1}<0$. Thus, as long as $\hat{w}_{2}(t)>\hat{w}_{1}(t)$,

$$
\hat{w}_{2}(t)-\hat{w}_{1}(t)<\Delta+M_{3} \gamma-\gamma M_{4} \int_{\hat{t}_{2}}^{t} \hat{w}_{2}-\hat{w}_{1} \mathrm{~d} \tau .
$$

We apply Gronwall's inequality to conclude that if $\gamma$ is sufficiently small, then

$$
\hat{w}_{2}(t)-\hat{w}_{1}(t) \leq\left(\Delta+M_{3} \gamma\right) \mathrm{e}^{-\gamma M_{4}\left(t-\hat{t}_{2}\right)} .
$$

We now consider the orientation reversing and preserving cases separately. Suppose that $(\mathrm{R})$ is satisfied and $\beta<v_{\theta}$ (see Fig. 9). Then both $\hat{w}_{i}(t)$ continue to increase with $\hat{w}_{2}(t)>\hat{w}_{1}(t)$ until $\hat{v}_{2}$ crosses $v_{\theta}$ when $t=\hat{T}_{2}$. The time $\hat{T}_{2}-\hat{t}_{2}$ is $\mathrm{O}(1 / \gamma)$ so there exists $\alpha \in(0,1)$, which does not depend on $\gamma$, such that $\exp \left\{-\gamma M_{4}\left(\hat{T}_{2}-\hat{t}_{2}\right)\right\}<\alpha$. From (3.5), we conclude that

$$
0<\hat{w}_{2}\left(\hat{T}_{2}\right)-\hat{w}_{1}\left(\hat{T}_{2}\right)<\alpha\left(\Delta+M_{3} \gamma\right) .
$$

For $t>\hat{T}_{2}, \hat{w}_{1}(t)$ continues to increase, at least until cell 1 crosses $v=v_{\theta}$ at $t=\hat{T}_{1}$. Moreover, $p_{1}(t)$ no longer receives input from cell 2, so $p_{1}(t)$ now satisfies (2.4). As discussed in Remark 5, this decreases the magnitude of the slope of $p_{1}(t)$. As in the proof of Lemma 3.2, this implies that $\hat{w}_{1}\left(\hat{T}_{2}\right)<\hat{w}_{1}\left(\hat{T}_{1}\right)<\hat{w}_{2}\left(\hat{T}_{2}\right)$. Hence, from (3.6)

$$
0<\hat{w}_{2}\left(\hat{T}_{2}\right)-\hat{w}_{1}\left(\hat{T}_{1}\right)<\alpha\left(\Delta+M_{3} \gamma\right) .
$$

Since each cell crosses $v=v_{\theta}$ exponentially close to $p_{i}(t)$, we can choose $\alpha$ and $M_{3}$ such that

$$
0<w_{2}\left(T_{2}\right)-w_{1}\left(T_{1}\right)<\alpha\left(\Delta+M_{3} \gamma\right) .
$$

By continuous dependence, a similar estimate holds if $(\mathrm{R})$ is satisfied with $v_{\theta}<\beta$ and $\beta-v_{\theta}$ is sufficiently small. 
After the cells cross $v_{\theta}$ they move horizontally to the left branch of $C$ and then along the left branch until one of the cells reaches the left knee. Along their evolution to the left knee, the $w$-values of the cells further compress, as another Gronwall-type argument shows. Hence,

$$
\Pi(\Delta)<\alpha\left(\Delta+M_{3} \gamma\right) .
$$

The proposition now follows if we let $M_{0}=\max \left\{M_{3}, \alpha M_{3} /(1-\alpha)\right\}$.

The argument for case $(\mathrm{P})$ is very similar, the only difference being that the $\hat{w}_{i}$ begin to decrease before the cells cross $v=v_{\theta}$. Choose $T_{D}$ so that $p_{2}(t)$ crosses the $w$-nullcline when $t=T_{D}$. Hence, $\hat{w}_{2}(t)$ reaches a maximum when $t=T_{D}$. Now $T_{D}-\hat{t}_{2}=\mathrm{O}(1 / \gamma)$, so there exists $\alpha_{1} \in(0,1)$ with $\exp \left\{-\gamma M_{4}\left(T_{D}-\hat{t}_{2}\right)\right\}<\alpha_{1}$. From (3.5), we conclude that

$$
0<\hat{w}_{2}\left(T_{D}\right)-\hat{w}_{1}\left(T_{D}\right)<\alpha_{1}\left(\Delta+M_{3} \gamma\right)
$$

Now, $\hat{w}_{2}(t)$ decreases for $T_{D}<t<\hat{T}_{2}$. Note that $\hat{T}_{2}-T_{D}=\mathrm{O}(1)$. This is because $v_{2}^{\prime}=\mathrm{O}(1)$ for $T_{D} \leq t \leq \hat{T}_{2}$ and $v_{D}-v_{\theta}=\mathrm{O}(1)$. Since $w_{2}^{\prime}=\mathrm{O}(\gamma)$, it follows that there must exist $M_{5}$ such that

$$
0<\hat{w}_{2}\left(T_{D}\right)-\hat{w}_{2}\left(\hat{T}_{2}\right)<M_{5} \gamma .
$$

Now $p_{1}(t)$ follows the same trajectory as $p_{2}(t)$ until $t=\hat{T}_{2}$. For $\hat{T}_{2} \leq t \leq \hat{T}_{1}, p_{1}(t)$ satisfies (2.4). As discussed in Remark 5, this decreases the magnitude of the slope of $p_{1}(t)$. It follows that $\hat{w}_{1}(t)$ increases to at most $\hat{w}_{2}\left(T_{D}\right)$ and then decreases until cell 1 crosses $v=v_{\theta}$. Hence,

$$
\left|\hat{w}_{2}\left(\hat{T}_{2}\right)-\hat{w}_{1}\left(\hat{T}_{1}\right)\right|<\left|\hat{w}_{2}\left(T_{D}\right)-\hat{w}_{2}\left(T_{2}\right)\right|+\left|\hat{w}_{2}\left(T_{D}\right)-\hat{w}_{1}\left(T_{D}\right)\right|<\alpha_{1} \Delta+\left(\alpha_{1} M_{3}+M_{5}\right) \gamma .
$$

Since each cell $i$ crosses $v=v_{\theta}$ exponentially close to $p_{i}(t)$, we can choose the constants so that the same estimate holds for $\left|w_{2}\left(T_{2}\right)-w_{1}\left(T_{1}\right)\right|$. The cells then move horizontally to the left branch of $C$ and then down that branch. As before, the $w$-values of the cells continue to compress. We therefore have that

$$
\Pi(\Delta)<\alpha_{1} \Delta+\left(\alpha_{1} M_{3}+M_{5}\right) \gamma .
$$

The proposition now follows if $M_{0}=\max \left\{M_{3},\left(\alpha_{1} M_{3}+M_{5}\right) /\left(1-\alpha_{1}\right)\right\}$.

Remark 7. The synchronizing effect for two cells starting at a large enough $\mathrm{O}(\gamma)$ distance is analogous to the synchronization associated with "fast threshold modulation" (FTM) for a pair of cells with only two time scales $\mathrm{O}(1)$ and $\mathrm{O}(\epsilon)$. In the FTM case, the synchrony arises because the $w$-coordinate does not change across a jump, while the rate in the active state is higher than that in the silent state, producing a compression in the time metric. In the current case, in which there is an intermediate time scale, there is a change in $w$ across the the jump; however, the dominant effect in the synchronization is still that the progress through the active state is much faster than that in the silent state.

\subsection{Contraction mapping argument}

We now produce an interval $\left(\Delta_{\exp }, \Delta_{\text {com }}\right)$ with the properties that $0<\Delta_{\exp }<\Delta_{\text {com }}, \Pi\left(\Delta_{\exp }\right)>\Delta_{\exp }, 0<$ $\Pi\left(\Delta_{\text {com }}\right)<\Delta_{\text {com }}$, and $\Pi$ is a contraction map on that interval. This will imply that the graph of $\Pi$ crosses the diagonal with slope strictly between -1 and +1 , and hence there is a unique fixed point in that interval.

From the expansion argument, we know that we can choose a $\Delta_{\exp }$ so that $\Pi\left(\Delta_{\exp }\right)>\Delta_{\exp }$. For $\Delta_{\text {com }}$, the contraction map argument becomes easier if we choose a value that is not too large. More specifically, consider the case (R). We know that for small enough $\Delta$, cells reverse their orientation after one cycle. However, for cells that start far enough apart, the compression argument for case $(\mathrm{R})$ showed that cells preserve their initial orientation. 


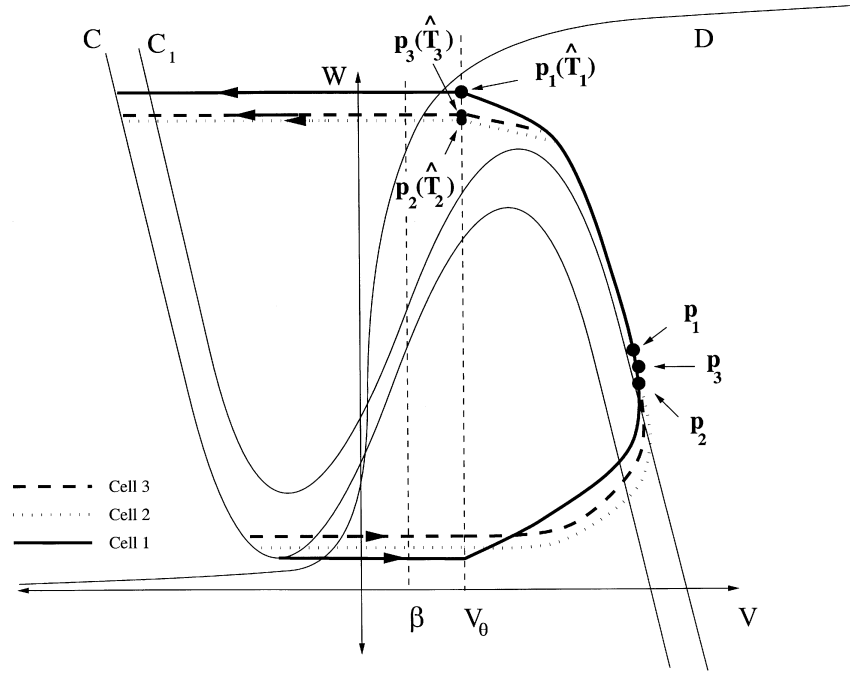

Fig. 10. The contraction of cells 2 and 3 for case (R). The ordering of times is $\hat{T}_{1}<\hat{T}_{3}<\hat{T}_{2}$. Throughout their trajectories, cells 2 and 3 are governed by the same sets of equations.

We choose $\Delta_{\text {com }}>\Delta_{\exp }$ so that on $\left[\Delta_{\exp }, \Delta_{\text {com }}\right.$, the map $\Pi$ reverses orientation after one cycle. Since there are points $\Delta$ at which $\Pi$ is orientation reversing, and others for which $\Pi$ is orientation preserving, $\Delta_{\text {com }}$ may be chosen so that $\Pi\left(\Delta_{\text {com }}\right)$ is arbitrarily close to 0 ; in particular, we may choose it so that $0<\Pi\left(\Delta_{\text {com }}\right)<$ $\Delta_{\text {com. }}$. A similar argument works for the case (P), but now the map $\Pi$ is orientation preserving on the interval of interest.

The argument that $\Pi$ is a uniform contraction map has a different flavor than the compression argument. In the latter, we considered a trajectory starting above the left knee and interacting with the one starting at that knee. To show that $\Pi$ is uniformly contracting, we must show that any two points $\Delta_{2}, \Delta_{3}$ in the interval $\left(\Delta_{\text {exp }}, \Delta_{\text {com }}\right)$ get brought closer together under П. For this, trajectories starting out above the knee each interact with the one starting at the knee, not with each other; that is, it is the trajectory starting at the knee whose position determines when the other trajectories receive excitation.

The following result demonstrates that $\Pi$ is a uniform contraction on $\left(\Delta_{\exp }, \Delta_{\text {com }}\right)$.

Proposition 3.3. There exists $\alpha_{2} \in(0,1)$ such that if $\Delta_{\exp }<\Delta_{2}<\Delta_{3}<\Delta_{\text {com }}$ and $\gamma$ is sufficiently small, then $\left|\Pi\left(\Delta_{3}\right)-\Pi\left(\Delta_{2}\right)\right|<\alpha_{2}\left(\Delta_{3}-\Delta_{2}\right)$.

Proof. As before, we follow the trajectories corresponding to each cell around in phase space. Cell 1 starts from the left-hand knee, and cells 2 and 3 start above it.

When cell 1 crosses $v_{\theta}$ it excites cells 2 and 3, thus causing them to jump up. Their trajectories are horizontal until $v_{\theta}$. Let $p_{i}=\left(\hat{v}_{i}, \hat{w}_{i}\right)$ be defined as before, now for $i=1,2,3$. Recall that these are the points on $R_{\gamma}$ which have the same asymptotic phase as cell $i$. Because of our choice of $\Delta_{\text {com }}$, cell 1 now has the largest $w$-value. The other two do not reverse orientation, so $\hat{w}_{1}>\hat{w}_{3}>\hat{w}_{2}$ (see Fig. 10).

We now need results concerning the Fenichel fibration [6]. These results state that the fibers depend smoothly on both $\gamma$ and their position along $R_{\gamma}$. Since the fibers are horizontal when $\gamma=0$, it follows that there exists $M_{6}$ such that, for $\gamma$ sufficiently small,

$$
\left|\hat{w}_{3}-\hat{w}_{2}\right| \leq\left(1+M_{6} \gamma\right)\left(\Delta_{3}-\Delta_{2}\right) .
$$


Let $\left(\hat{v}_{i}(t), \hat{w}_{i}(t)\right)$ be the solution of (2.6) which passes through $p_{i}$ at $t=\hat{t}_{i}$. A Gronwall-type argument, just as in the preceding section, shows that as long as $\hat{w}_{3}(t)>\hat{w}_{2}(t)$,

$$
\hat{w}_{3}(t)-\hat{w}_{2}(t)<\left(\hat{w}_{3}-\hat{w}_{2}\right) \mathrm{e}^{-\gamma M_{7}\left(t-\hat{t}_{3}\right)}<\left(1+M_{6} \gamma\right)\left(\Delta_{3}-\Delta_{2}\right) \mathrm{e}^{-\gamma M_{7}\left(t-\hat{t}_{3}\right)},
$$

for some $M_{7}$.

Now $p_{1}(t)$ is the first to cross $v_{\theta}$. If $(\mathrm{R})$ is satisfied, then $\hat{w}_{2}$ and $\hat{w}_{3}$ continue to increase until $p_{2}(t)$ and $p_{3}(t)$ cross $v_{\theta}$, say when $t=\hat{T}_{i}$. Hence, (3.7) continues to hold. The time for these cells to cross $v_{\theta}$ is $\mathrm{O}(1 / \gamma)$, so as before, there exists $\alpha_{2} \in(0,1)$ such that

$$
\left|\hat{w}_{3}\left(\hat{T}_{3}\right)-\hat{w}_{2}\left(\hat{T}_{2}\right)\right|<\alpha_{2}\left(1+M_{6} \gamma\right)\left(\Delta_{3}-\Delta_{2}\right) .
$$

A similar estimate must also hold for $\left|w_{3}\left(T_{3}\right)-w_{2}\left(T_{2}\right)\right|$.

After the cells cross $v_{\theta}$, they approach the left branch of $C$ horizontally and then proceed down this left branch. A Gronwall-type argument shows that during this time, cells 2 and 3 are further compressed. Since $\alpha_{2}<1$, the proposition follows from (3.8) if $\gamma$ is sufficiently small.

A similar argument holds if (P) is satisfied. Just as in the proof of Proposition 3.2, an additional estimate is needed because the $\hat{w}_{i}(t)$ begin to decrease before the cells reach the threshold at $v_{\theta}$. We do not give the details here, since the analysis is so similar to that done previously.

\section{Proof of Theorem 2}

We demonstrate in this section that Theorem 2 follows from Theorem 1 and Theorem 1.2 in [10]. In order to explain why this is so, we need to view solutions of the model from a more traditional dynamical systems point of view. We have so far imagined the solution as two points, each corresponding to one of the oscillators, evolving in a two-dimensional phase space. We now imagine the solution as a single point evolving in the full four-dimensional phase space.

We need to reinterpret the geometric constructions of the singular solutions described earlier. First consider the silent phase. Before we considered two points $\left(v_{1}, w_{1}\right)$ and $\left(v_{2}, w_{2}\right)$, evolving along the one-dimensional left branch of $C$ until one of the points reached the left knee of $C$. We now think of this as a single trajectory moving along a two-dimensional slow manifold until the trajectory reaches a fold, or curve of knees, of that slow manifold. The slow manifold is given by

$$
\mathcal{M} \equiv\left\{\left(v_{1}, v_{2}, w_{1}, w_{2}\right): v_{1}=v_{\mathrm{L}}\left(w_{1}\right), v_{2}=v_{\mathrm{L}}\left(w_{2}\right), w_{1}>0, w_{2}>0\right\},
$$

and its fold is given by

$$
\mathcal{F} \equiv \mathcal{M} \cap\left\{w_{1}=0 \text { or } w_{2}=0\right\} .
$$

Here we have assumed that $w_{\mathrm{LK}}=0$, as before.

We actually have to consider only the evolution of the slow variables $w_{1}$ and $w_{2}$. This is because each of the fast variables is given by $v_{i}=v_{\mathrm{L}}\left(w_{i}\right)$. Hence, we can project the full trajectory, along with the slow manifold $\mathcal{M}$ and the fold $\mathcal{F}$, onto the $\left(w_{1}, w_{2}\right)$ slow phase plane. This reduces the full system to the following two-dimensional system for the slow variables:

$$
w_{1}^{\prime}=g\left(v_{\mathrm{L}}\left(w_{1}\right), w_{1}\right), \quad w_{2}^{\prime}=g\left(v_{\mathrm{L}}\left(w_{2}\right), w_{2}\right) .
$$

This system remains valid as long as

$$
\left(w_{1}, w_{2}\right) \in \mathcal{M}_{\mathrm{P}} \equiv\left\{\left(w_{1}, w_{2}\right): w_{1}>0 \text { and } w_{2}>0\right\} .
$$


The silent phase ends when $\left(w_{1}, w_{2}\right)$ reaches the boundary of $\mathcal{M}_{\mathrm{P}}$ along the curve

$$
\mathcal{F}_{\mathrm{P}} \equiv\left\{\left(w_{1}, w_{2}\right): \text { either } w_{1}=0 \text { and } w_{2} \geq 0 \text {, or } w_{1} \geq 0 \text { and } w_{2}=0\right\} .
$$

Note that $\mathcal{F}_{\mathrm{P}}$ is smooth except there is a 'corner' at the origin; this corresponds to the point where the synchronous solution leaves the silent phase.

Now the return map $\Pi$ can be viewed as defining a map from some subset of $\mathcal{F}_{\mathrm{P}}$ back into $\mathcal{F}_{\mathrm{P}}$. We will also denote this map as $\Pi$. Theorem 1 states that if $(\mathrm{P})$ or $(\mathrm{R})$ is satisfied and $\gamma$ is sufficiently small, then $\Pi$ is a uniform contraction on a subset of $\mathcal{F}_{\mathrm{P}}$ that is an $\tilde{\mathrm{O}}(\gamma)$ distance away from the origin. This subset contains the fixed point corresponding to the almost-synchronous singular solution. As a result, there is no problem in showing that this map is differentiable in an $\mathrm{O}(\gamma)$ neighborhood of the fixed point. So we conclude that the linearization of $\Pi$ at its fixed point has one eigenvalue which has absolute value less than one.

We now wish to invoke Theorem 1.2 in [10] to conclude that the singular periodic solution given by Theorem 1 perturbs, for $\epsilon>0$, to an asymptotically stable $\mathrm{O}(\epsilon)$-solution of (2.2). To do this, we must check that the hypotheses of the theorem in [10] are satisfied. These are considered below. In what follows, we fix $\gamma$ so that the conclusion of Theorem 1 holds. We also assume that the fixed point of $\Pi$, given by Theorem 1 , is at $w_{2}=\Delta$. Hence, if we view $\Pi$ as a map from a subset of $\mathcal{F}_{\mathrm{P}}$ into $\mathcal{F}_{\mathrm{P}}$ then the fixed point is at $\left(w_{1}, w_{2}\right)=(0, \Delta) \equiv p_{0}$. Of course, by symmetry, there is also a fixed point at $(\Delta, 0)$.

The first hypothesis that we need to verify concerns solutions of the slow subsystem (2.5). We must check that the solution of (4.1) corresponding to the singular periodic solution approaches $p_{0}$ transverse to $\mathcal{F}_{\mathrm{P}}$. However, this is trivial since at $p_{0}$, both $w_{1}^{\prime}$ and $w_{2}^{\prime}$ are non-zero.

The second hypothesis is concerned with the fast subsystem. Consider the point $P_{0}=\left(v_{1}, v_{2}, w_{1}, w_{2}\right)=$ $\left(v_{\mathrm{L}}(0), v_{\mathrm{L}}(\Delta), 0, \Delta\right)$. This is the point in the full phase space that lies on the singular periodic solution along the fold $\mathcal{F}$ in $\mathcal{M}$. That is, $p_{0}$ is the projection of $P_{0}$ onto the slow phase plane. We must show that there is a unique solution of the fast subsystem which approaches $P_{0}$ as $t \rightarrow-\infty$. This will be the case if the left knee of $C$ is in some sense non-degenerate. That is, if we write the cubic $C$ as $w=h(v)$, then $h " \neq 0$ at the left knee of $C$. We assume that this is the case.

The next hypothesis that has to be verified is concerned with the map П. We need precisely what was discussed above; that is, the linearization of $\Pi$ has all of its eigenvalues inside of the unit circle.

We also need that the jump-up curve $\mathcal{F}_{\mathrm{P}}$ is smooth. This is certainly true at $p_{0}$. Note that this is not the case at the origin where $\mathcal{F}_{\mathrm{P}}$ has a corner. Hence, one cannot apply the results in [10] directly to analyze the stability properties of the synchronous solution.

There is one more technical condition to worry about. In order to apply the results of [10], we need that the vector field defined by the model is sufficiently smooth. This is actually not the case because of the Heaviside step functions. Once we prove Theorem 1, however, we can smooth out the discontinuities in the Heaviside functions to conclude that Theorem 1 is still valid for the smooth approximations. We are then justified to apply the results in [10].

\section{Numerical simulations}

We simulated the network of two coupled cells using the equations given in Appendix A. In Fig. 11, we show voltage traces of an order preserving solution. The parameters $\epsilon=0.001, \gamma=0.025, v_{\theta}=v_{\mathrm{st}}=-15, v_{3}=$ -5 and $g_{\text {syn }}=0.5$ were used. The parameter $\beta$ which appears in the analysis does not enter in the simulations. Instead the parameter $v_{3}$, which is the half activation voltage for an outward current associated with the $g$-nullcline, does. If the rise of the $g$-nullcline is sufficiently sharp, then $v_{3}$ closely approximates $\beta$. Notice that 


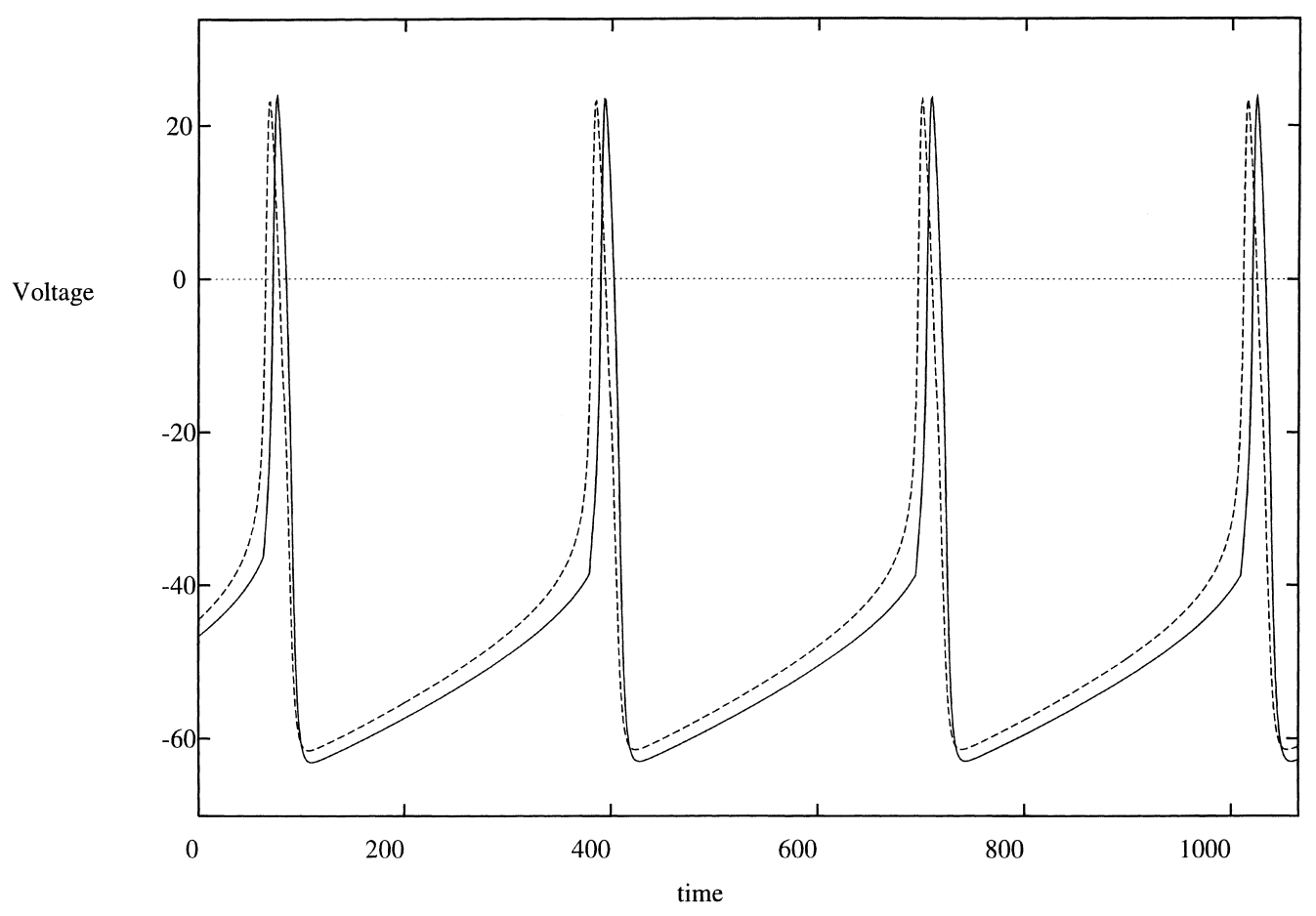

Fig. 11. Order preserving solution. Time is measured in ms.

$v_{\theta}<v_{3}$ produces an order preserving orbit which is consistent with condition $(\mathrm{P})$ of Theorem 1. By changing the parameters to $v_{\theta}=v_{\mathrm{st}}=0, v_{3}=-20$, and $g_{\mathrm{syn}}=2.0$, we obtain an order reversing solution, depicted in Fig. 12. Again note that the choice of these parameters falls into condition (R) for which the solution is order reversing.

In our analysis, we showed how $\mathrm{O}(\gamma)$ differences in the $w$-values of the cells as they evolve along the left branch of $C$ get transformed into $\mathrm{O}(1)$ differences in the time at which they cross the threshold $v_{\theta}$. In Table 1, we verify this fact and show how the relative lag time at $v_{\theta}$ is a fraction of the period of the cell. In the table, the lag time is computed by constructing a Poincare section at the position of cell 1 , at for example $v=-50 \mathrm{mV}$, and measuring the time cell 2 takes to reach this section. The relative lag at $v=v_{\theta}$ is simply the lag at $v_{\theta}$ divided by the period of the cell. The parameters are the same as in Fig. 11.

Our analysis concentrated only on the existence of order reversing and preserving orbits. Other solutions do exist, however. When the initial conditions are outside the basin of attraction of the $\mathrm{O}(\epsilon)$-solution then the cells may be attracted to an anti-phase orbit as shown in Fig. 13. Here the parameters are identical to Fig. 11. Rigorous conditions and proofs for existence of anti-phase solutions in relaxation oscillator models are given in [9]. This study reports that there exists a region of bistability in parameter space in which both the anti-phase and the synchronous solution are stable. For our case, the simulations indicate bistability between an anti-phase and $\mathrm{O}(\epsilon)$-synchronous solution. However, since the duty cycle of our cells, the ratio of time in the active phase to time in the silent phase, is $\mathrm{O}(\epsilon / \gamma)$ and therefore small, initial conditions which start away from the anti-phase solution take long amounts of time to actually approach it. This is consistent with results in [9] which show that the time length of transients is inversely related to the duty cycle. 


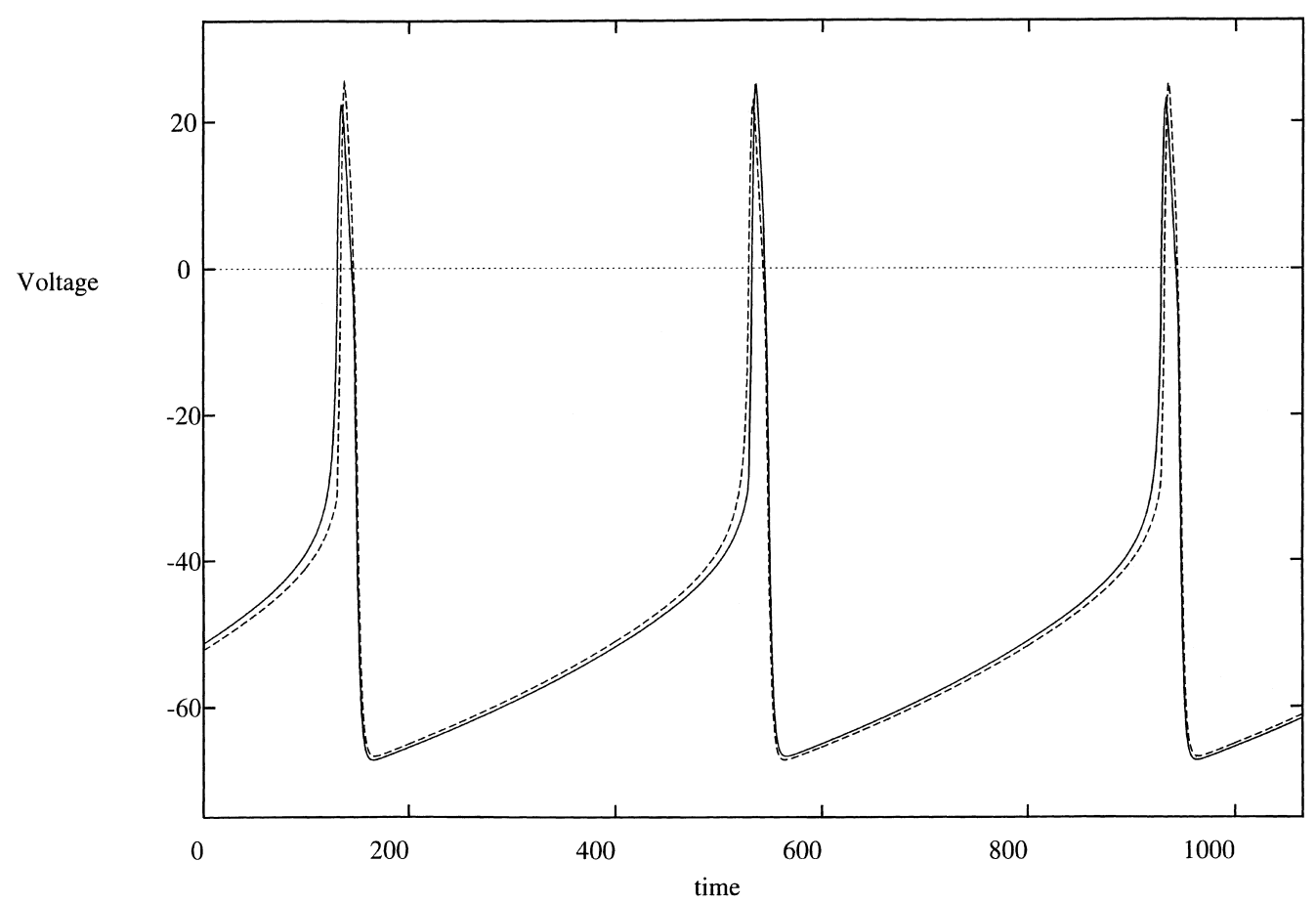

Fig. 12. Order reversing solution. Time is measured in ms.

In our analysis, we restricted the parameters to $v_{\theta}=v_{\text {st }}$. In this situation, the synchronous solution is unstable. By changing $v_{\theta}>v_{\text {st }}$, we can stabilize the synchronous solution. In Fig. 14, we start with the parameters of the order preserving orbit of Fig. 11. At $t=500 \mathrm{~ms}$, we change $v_{\theta}=-5$, and the cells quickly synchronize. At $t=1200 \mathrm{~ms}$, we change back to $v_{\theta}=-15$ to illustrate the instability of the synchronous solution when $v_{\theta}=v_{\mathrm{st}}$. The reason why changing $v_{\theta}>v_{\mathrm{st}}$ affects the stability of solutions is straightforward. Consider $\epsilon=0$, and let $v_{\theta}>v_{\mathrm{st}}$ independent of their relationship to $\beta$. When cell 1 jumps up, it will cross $v_{\text {st }}$ before it reaches $v_{\theta}$, causing cell 2 to jump up. However, until it reaches $v_{\theta}$, cell 1 will still be governed by (2.3), where $w^{\prime}=0$. This limits the expansion on the up jump. In fact, analysis similar to that given in Lemma 3.1 demonstrates that the range of voltages over which expansion takes place is $\left(v_{\theta}, v_{F}\right)$. Hence, if $v_{\theta}>v_{F}$, then no expansion will take place and the synchronous solution will be stable. Recall, from the proof of Lemma 3.1, that $v_{F}$ is determined by the time it takes for cell 2 to cross $v_{\mathrm{st}}$. Hence, raising $v_{\theta}$ and lowering $v_{\mathrm{st}}$ both help to stabilize the synchronous solution.

Table 1

The table shows how differences in the $w$-values of the cells at $v=-50 \mathrm{mV}$ get transformed into differences in times to threshold $v_{\theta}$

\begin{tabular}{|c|c|c|c|c|c|}
\hline$\gamma$ & Period (ms) & Lag at $V=-50(\mathrm{~ms})$ & $w_{2}-w_{1}$ at $V=-50$ & Lag at $v=v_{\theta}(\mathrm{ms})$ & Relative lag at $V=v_{\theta}$ \\
\hline 0.001 & 573 & 0 & 0 & 0 & 0 \\
\hline 0.005 & 388 & 7 & 0.007 & 5 & 0.012 \\
\hline 0.01 & 360 & 10 & 0.010 & 5.5 & 0.015 \\
\hline 0.02 & 332 & 19 & 0.019 & 8 & 0.024 \\
\hline 0.025 & 315 & 32 & 0.032 & 9 & 0.028 \\
\hline
\end{tabular}




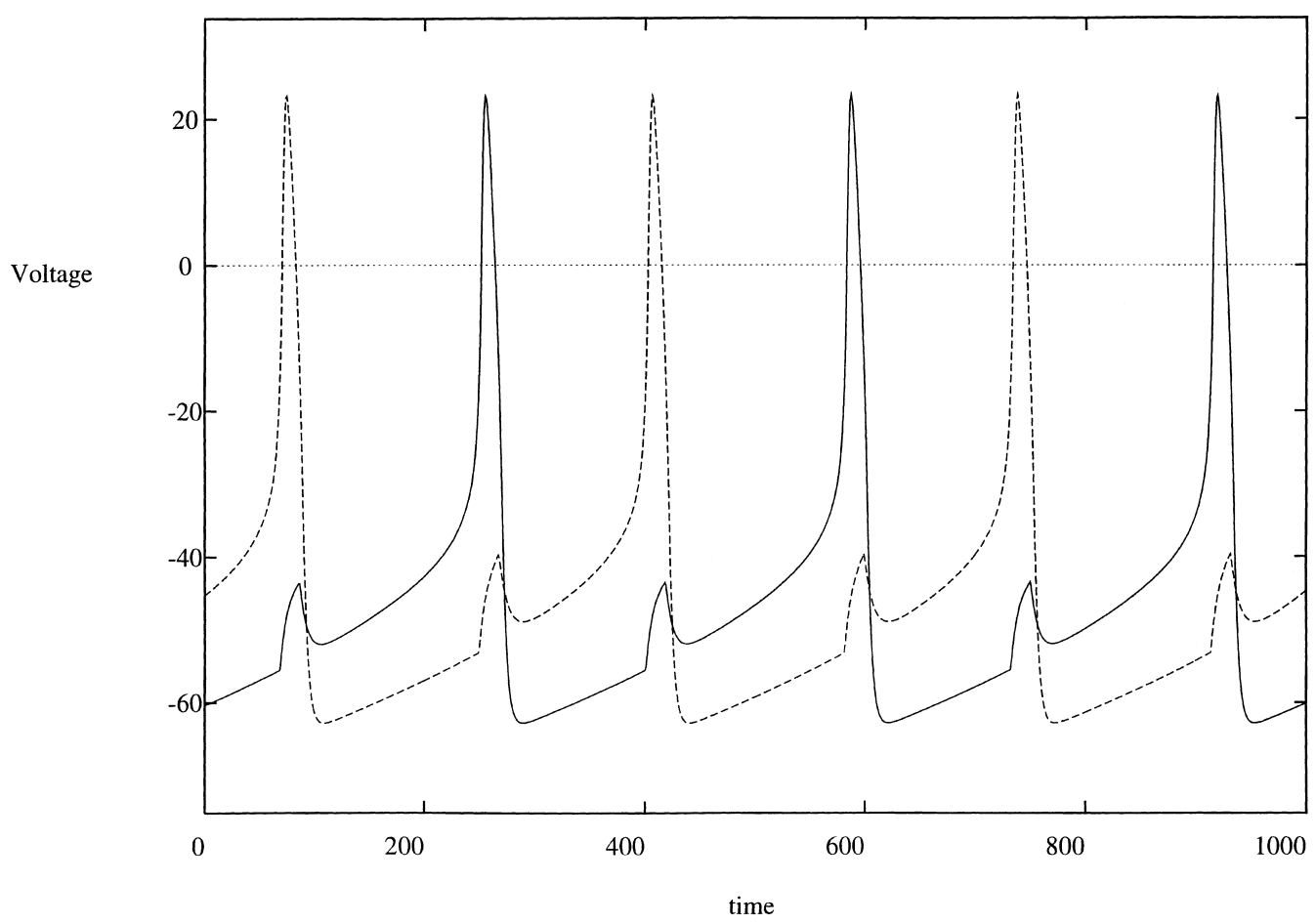

Fig. 13. Anti-phase solution. Time is measured in ms.

By taking $v_{\theta}<v_{\text {st }}$ and specifying that both lie to one side or the other of $v_{3}$ (or $\beta$ ) one can enhance the time lag between cells in either the order reversing or preserving case. The logic is similar to above. This change expands the region in phase space over which the cells are governed by different equations, thus heightening the amount of expansion. When the parameter $\beta$ lies between $v_{\theta}$ and $v_{\mathrm{st}}$ the situation is more complicated.

\section{Discussion}

In this paper, we have shown that two cells coupled by instantaneous excitatory synapses can display a variety of firing patterns ranging from almost-synchronous to synchronous to anti-phase solutions. The existence and stability of these different solutions depend critically on the relative values of the thresholds $v_{\theta}$, which is intrinsic to the cell, and $v_{\mathrm{st}}$, which is related to the synapses between cells. For the almost-synchronous solution, the effect of the intermediate time scale is to provide a region in phase space over which the cells follow different differential equations. This difference in governing equations creates an expansion for cells that are too close together and a compression for cells which are too far apart. Our analysis provides bounds for not only the degree of asynchrony in the almost-synchronous solution, but also the basin of attraction of this solution.

Many of the works studying cells coupled by excitation $[1,7,8,21,22]$ use simplified description of the cells such as integrate and fire or spiking neuron models. These studies report that the synchronous solution is stable if the rise time of excitation is fast enough. In our work, using instantaneous synapses which have infinitely fast excitatory rise times, we have shown that the synchronous solution is unstable, and that a nearby almost-synchronous solution is in fact stable. One reason for the difference in our conclusions is that these simplified models ignore the dynamics of 


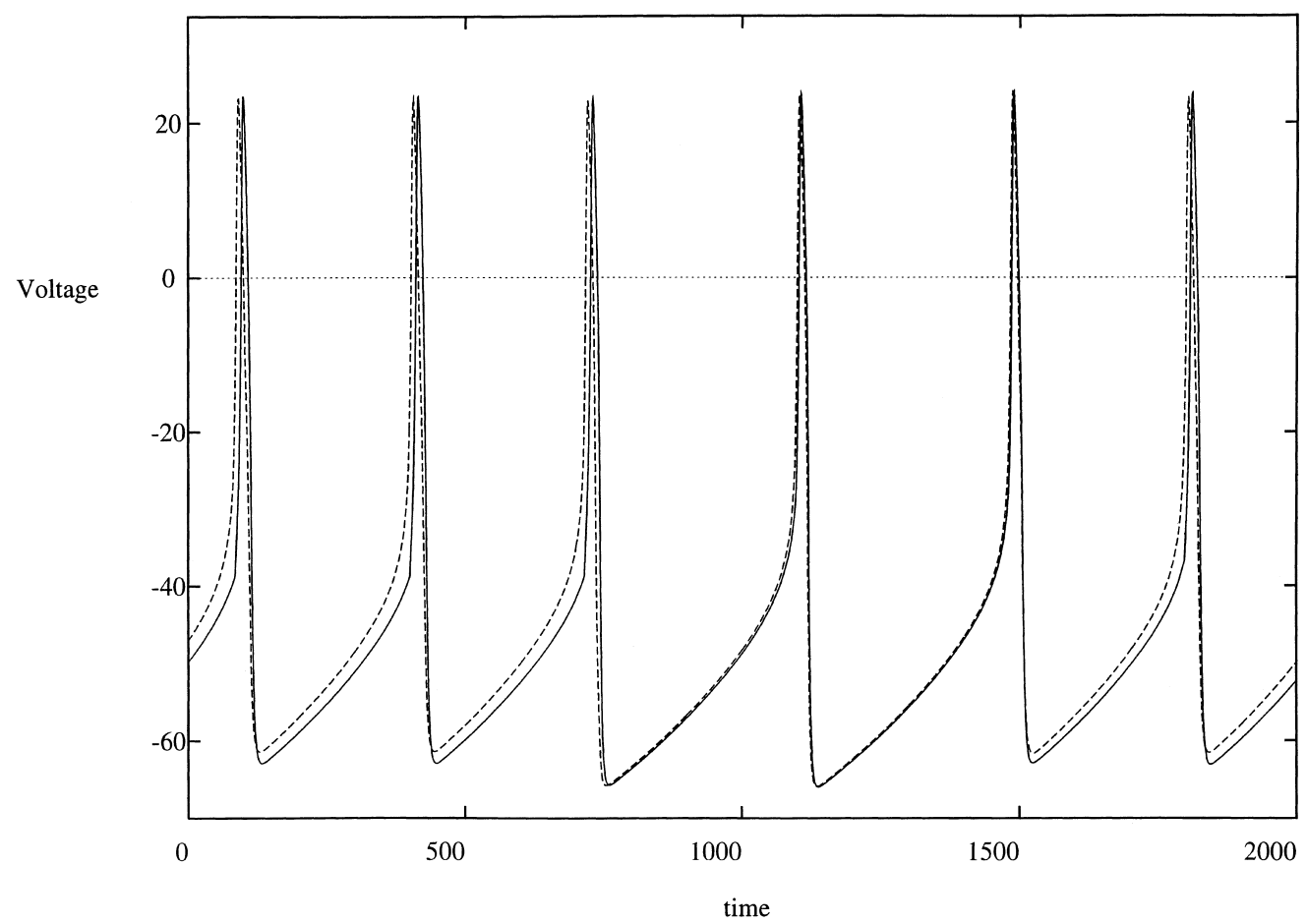

Fig. 14. Effects of making $v_{\theta}>v_{\mathrm{st}}$. The cells were started with the order preserving parameters of Fig. 11 with $v_{\theta}=v_{\mathrm{st}}$. At $t=500 \mathrm{~ms}$, we changed to $v_{\theta}=-5$. At $t=1200 \mathrm{~ms}$, we changed back to $v_{\theta}=-15$. The figure shows how the thresholds $v_{\theta}$ and $v_{\text {st }}$ can modulate the stability of the synchronous solution.

cells during their active phase; we have shown that the dynamics of the active phase of a cell can determine whether or not synchrony is stable, as well as the amount of asynchrony that may occur.

Almost-synchronous solutions can also arise in networks with excitatory synapses if there is some heterogeneity in either the individual cells or the total amount of synaptic input which individual cells receive $[12,17,19]$. In this paper, we assumed that the network is completely homogeneous. The almost-synchronous solution arises because of interactions between different time scales, not because of heterogeneities in the network. As discussed above, the almost-synchronous solution considered here is created primarily while the cells are in the active phase and satisfy different differential equations. Unlike [12], we were able to give specific conditions on the biophysical parameters insuring that almost-synchrony occurs. We also considered relatively strong coupling between the cells in the active phase, unlike [12].

Several papers $[8,9,16,22]$ report that stable anti-phase solutions may exist in the same parameter ranges as the synchronous solution. Our numerical simulations support this conclusion except that the bistability may also be between the anti-phase and almost-synchronous solutions. Note that in $[9,16]$, the intrinsic dynamics of the cells occur on only two time scales, precluding the possibility of almost-synchronous solutions.

The model of a cell with three time scales that we have described may provide certain computational advantages. Suppose, for instance, that a downstream detector receives input from the network described above. If the detector measures firing rate, then it will be unable to distinguish between an almost-synchronous and synchronous solution. In this case, the cells may as well be governed by standard two-time scale models. However, if the downstream detector measures inter-spike interval, then it will be able to discriminate between almost-synchronous and 
synchronous solutions. In this case, our three-time scale model possesses the advantage that by modulating various thresholds, it can produce stable behaviors of both of these types. Moreover, by adjusting parameters in the model, the degree of asynchrony, or alternatively, the inter-spike interval, can be varied. Thus these neurons are capable of encoding far more information than two-time scale cells.

\section{Appendix A}

The equations that we used are based on the Morris-Lecar equations [11], with parameter values largely taken from [14]. Only those that were fixed throughout the simulations are listed below. Those parameters that were varied are given in Section 5. The equations for each oscillator, for $i \neq j$, are

$$
\begin{aligned}
& v_{i}^{\prime}=\frac{\left(-g_{l}\left(v_{i}-v_{l}\right)-g_{k} w_{i}\left(v_{i}-v_{k}\right)-g_{\mathrm{ca}} m_{\infty}\left(v_{i}\right)\left(v_{i}-v_{\mathrm{ca}}\right)-g_{\mathrm{syn}} H\left(v_{j}-v_{\mathrm{st}}\right)\left(v_{i}-v_{\mathrm{syn}}\right)-I_{\mathrm{app}}\right)}{c}, \\
& w_{i}^{\prime}=\frac{\epsilon\left(w_{\infty}\left(v_{i}\right)-w_{i}\right)}{\tau_{\infty}\left(v_{i}\right)},
\end{aligned}
$$

where

$$
\begin{aligned}
& m_{\infty}(v)=0.5\left(1.0+\tanh \left(\frac{v-v 1}{v 2}\right)\right), \quad w_{\infty}(v)=0.5\left(1.0+\tanh \left(\frac{v-v 3}{v 4}\right)\right), \\
& \tau_{\infty}(v)=0.5\left(1.0+\tanh \left(\frac{v-v_{\theta}}{v_{r}}\right)\right)\left(\frac{\epsilon}{\gamma}-1.0\right)+1.0 .
\end{aligned}
$$

The parameters $g_{\mathrm{ca}}=5, v_{1}=-10, v_{2}=18, g_{k}=8, v_{4}=4, g_{l}=2, I_{\mathrm{app}}=90, c=100, v_{\mathrm{ca}}=120, v_{k}=-84$, $v_{l}=-60, v_{\mathrm{syn}}=40$, and $v_{r}=0.001$. The equations were scaled by a factor of 4 to speed up the oscillations since $\epsilon$ is taken to be so small.

\section{References}

[1] L. Abbot, C. van Vreeswijk, Asynchronous states in networks of pulsecoupled oscillators, Phys. Rev. E 48 (1993) $1483-1490$.

[2] C. Chow, Phase-locking in weakly heterogeneous neuronal networks, Phys. D 118 (1998) 343-370.

[3] S.M. Crook, G.B. Ermentrout, M. Vanier, J. Bower, The role of axonal delays in the synchronization of networks of coupled cortical oscillators, J. Comp. Neurol. 4 (1997) 161-172.

[4] S.M. Crook, G.B. Ermentrout, J.M. Bower, Dendritic and synaptic effects in systems of coupled cortical oscillators, J. Comp. Neurol. 5 (1998) 315-329.

[5] S.M. Crook, G.B. Ermentrout, J.M. Bower, Spike frequency adaptation affects the synchronization properties of networks of cortical oscillators, Neur. Comp. 10 (1998) 837-854.

[6] N. Fenichel, Geometric singular perturbation theory for ordinary differential equations, J. Differential Equations 31 (1) (1979) 53-98.

[7] W. Gerstner, J.L. van Hemmen, J. Cowan, What matters in neuronal locking, Neur. Comp. 8 (1996) 1653-1676.

[8] D. Hansel, G. Mato, C. Meunier, Synchrony in excitatory neural networks, Neur. Comp. 7 (1995) $307-337$.

[9] N. Kopell, D. Somers, Anti-phase solutions in relaxation oscillators coupled through excitatory interactions, J. Math. Biol. 33 (1995) 261-280.

[10] E.F. Mishchenko, Yu.S. Koselev, A.Yu. Koselev, N.Kh. Rozov, Asymptotic methods in singularly perturbed systems, in: R. Gamkrelidze (Ed.), Monographs in Contemporary Mathematics, Consultants Bureau, New York, 1994.

[11] C. Morris, H. Lecar, Voltage oscillations in the barnacle giant muscle fiber, Biophys. J. 35 (1981) $193-213$.

[12] P. Pinsky, Synchrony and clustering in an excitatory neural network model with intrinsic relaxation kinetics, SIAM J. Appl. Math. 55 (1995) 220-241.

[13] P. Pinsky, J. Rinzel, Intrinsic and network rhythmogenesis in a reduced Traub model for CA3 neurons, J. Comp. Neurol. 1 (1994) 39-60.

[14] J. Rinzel, G.B. Ermentrout, Analysis of neural excitability and oscillations, in: C. Koch, I. Segev (Eds.), Methods in Neuronal Modeling: From Synapses to Networks, MIT Press, Cambridge, MA, 1997, pp. 135-170. 
[15] J. Rubin, D. Terman, Geometric analysis of population rhythms in synaptically coupled neuronal networks, Neur. Comp., in press.

[16] D. Somers, N. Kopell, Rapid synchronization through fast threshold modulation, Biol. Cyber. 68 (1993) 393-407.

[17] D. Somers, N. Kopell, Waves and synchrony in arrays of oscillators of relaxation and non-relaxation type, Phys. D 89 (1995) $169-183$.

[18] D. Terman, N. Kopell, A. Bose, Dynamics of two mutually coupled slow inhibitory neurons, Phys. D 117 (1998) 241-275.

[19] D. Terman, D. Wang, Global competition and local cooperation in a network of neural oscillators, Phys. D 81 (1995) 148-176.

[20] R. Traub, R. Miles, Neuronal Networks of the Hippocampus, Cambridge University Press, Cambridge, 1991.

[21] C. van Vreeswijk, Partial synchronization in populations of pulse-coupled oscillators, Phys. Rev. E 54 (1996) 5522-5537.

[22] C. van Vreeswijk, L. Abbott, G.B. Ermentrout, When inhibition, not excitation synchronizes neural firing, J. Comp. Neurosci. 1 (1994) 313-321.

[23] X.J. Wang, J. Rinzel, Alternating and synchronous rhythms in reciprocally inhibitory neurons, Neur. Comp. 4 (1992) 84-97. 